\title{
Integral Transforms and Bayesian Inference in the Identification of Variable Thermal Conductivity in Two-Phase Dispersed Systems
}

\author{
Carolina P. Naveira-Cotta , Helcio R. B. Orlande \& Renato M. Cotta
}

To cite this article: Carolina P. Naveira-Cotta, Helcio R. B. Orlande \& Renato M. Cotta (2010) Integral Transforms and Bayesian Inference in the Identification of Variable Thermal Conductivity in Two-Phase Dispersed Systems, Numerical Heat Transfer, Part B: Fundamentals, 57:3, 173-202, DOI: $10.1080 / 10407791003685106$

To link to this article: https://doi.org/10.1080/10407791003685106

\section{曲 Published online: 04 May 2010.}

\section{Submit your article to this journal $\llbracket$}

Џ Article views: 132

Citing articles: 12 View citing articles $[7$ 


\title{
INTEGRAL TRANSFORMS AND BAYESIAN INFERENCE IN THE IDENTIFICATION OF VARIABLE THERMAL CONDUCTIVITY IN TWO-PHASE DISPERSED SYSTEMS
}

\author{
Carolina P. Naveira-Cotta, Helcio R. B. Orlande, and \\ Renato M. Cotta \\ Laboratory of Transmission and Technology of Heat, Mechanical \\ Engineering Department, Universidade Federal do Rio de Janeiro, \\ Rio de Janeiro, Brazil
}

This work illustrates the use of Bayesian inference in the estimation of spatially variable thermal conductivity for one-dimensional heat conduction in heterogeneous media, such as particle-filled composites and other two-phase dispersed systems, by employing a Markov chain Monte Carlo (MCMC) method, through the implementation of the MetropolisHastings algorithm. The direct problem solution is obtained analytically via integral transforms, and the related eigenvalue problem is solved by the generalized integral transform technique (GITT), offering a fast, precise, and robust solution for the transient temperature field, which are desirable features for the implementation of the inverse analysis. Instead of seeking the function estimation in the form of a sequence of local values for the thermal conductivity, an alternative approach is proposed here, which is based on the eigenfunction expansion of the thermal conductivity itself. Then, the unknown parameters become the corresponding series coefficients. Simulated temperatures obtained via integral transforms are used in the inverse analysis. From the prescription of the concentration distribution of the dispersed phase, available correlations for the thermal conductivity are employed to produce the simulated results with high precision in the direct problem solution, while eigenfunction expansions with reduced number of terms are employed in the inverse analysis itself, in order to avoid the so-called inverse crime. Both Gaussian and noninformative uniform distributions were used as priors for comparison purposes. In addition, alternative correlations for the thermal conductivity that yield different predictions are also employed as Gaussian priors for the algorithm in order to test the inverse analysis robustness.

\section{INTRODUCTION}

Composite materials, consisting of a dispersed reinforcement phase embedded in a bulk matrix phase, have been providing engineers with increased opportunities for tailoring structures to meet a variety of property and performance requirements.

Received 9 September 2009; accepted 15 January 2010.

The authors would like to acknowledge the financial support provided by CNPq, CAPES, and FAPERJ, Brazilian agencies for the fostering of science. The authors are also deeply grateful to the reviewers for the very careful revisions and helpful suggestions.

Address correspondence to Renato M. Cotta, Laboratory of Transmission and Technology of Heat, Mechanical Engineering Department, POLI/COPPE, Universidade Federal do Rio de Janeiro, C.P. 68503, Rio de Janeiro 21945-970, Brasil. E-mail: cotta@mecanica.coppe.ufrj.br 


\section{NOMENCLATURE}

\begin{tabular}{|c|c|c|c|}
\hline$d(x)$ & linear dissipation operator coefficient, & $T$ & dimensionless temperature (potential) \\
\hline & Eq. $(1 a)$ & $\mathbf{T}$ & vector of estimated temperatures \\
\hline$f(x)$ & initial condition, Eq. $(1 b)$ & $w(x)$ & transient operator coefficient \\
\hline$k_{d}$ & $\begin{array}{l}\text { thermal conductivity of the filler } \\
\text { (dispersed phase) }\end{array}$ & & $\begin{array}{l}\text { (dimensionless thermal capacity), } \\
\text { Eq. (1a) }\end{array}$ \\
\hline $\begin{array}{l}k_{m} \\
k(x)\end{array}$ & $\begin{array}{l}\text { thermal conductivity of the matrix } \\
\text { diffusion operator coefficient }\end{array}$ & $\mathbf{W}$ & $\begin{array}{l}\text { covariance matrix of the measurement } \\
\text { errors }\end{array}$ \\
\hline & $\begin{array}{l}\text { (dimensionless thermal conductivity), } \\
\text { Eq. ( } 1 a)\end{array}$ & $\begin{array}{l}x \\
\mathbf{Y}\end{array}$ & $\begin{array}{l}\text { dimensionless longitudinal coordinate } \\
\text { vector of measurements }\end{array}$ \\
\hline $\begin{array}{l}k_{f}(x) \\
L\end{array}$ & $\begin{array}{l}\text { filter for thermal conductivity relation } \\
\text { dimensionless domain length }\end{array}$ & $\alpha_{\mathrm{k}}, \beta_{\mathrm{k}}$ & $\begin{array}{l}\text { boundary condition coefficients, } \\
\text { Eqs. }(1 c, d)\end{array}$ \\
\hline$M$ & $\begin{array}{l}\text { truncation order in eigenvalue problem } \\
\text { expansion }\end{array}$ & $\gamma$ & $\begin{array}{l}\text { parameter in filler concentration } \\
\text { variation }\end{array}$ \\
\hline$N$ & $\begin{array}{l}\text { truncation order in temperature } \\
\text { expansion }\end{array}$ & $\delta$ & $\begin{array}{l}\text { function in filler concentration } \\
\text { variation }\end{array}$ \\
\hline$N_{k}$ & truncation order in coefficients & $\lambda$ & eigenvalues of the auxiliary problem \\
\hline & expansion & $\mu$ & eigenvalues of the original problem \\
\hline$N_{i}$ & normalization integrals & $\phi(x)$ & filler concentration distribution, \\
\hline$N_{m}$ & total number of measurements & & Eqs. (22) \\
\hline$N_{P}$ & $\begin{array}{l}\text { number of parameters to be estimated, } \\
\text { Eq. }(28 a)\end{array}$ & $\begin{array}{l}\phi_{k}(t) \\
\psi\end{array}$ & $\begin{array}{l}\text { source term, Eqs. }(1 c, d) \\
\text { eigenfunctions of the original problem }\end{array}$ \\
\hline$N_{t}$ & number of measurements in time & $\Omega$ & eigenfunctions of the auxiliary problem \\
\hline$N_{x}$ & $\begin{array}{l}\text { number of measurements along the } \\
\text { spatial domain }\end{array}$ & Subscri & ts and Superscripts \\
\hline $\mathbf{P}$ & vector of unknown parameters & $i, n, m$ & order of eigenquantities \\
\hline$P(x, t)$ & source term, Eq. $(1 a)$ & - & integral transform \\
\hline$t$ & dimensionless time variable & $\sim$ & normalized eigenfunction \\
\hline
\end{tabular}

In recent years, various applications have been requiring composites with higher thermal conductivity. For instance, polymer composites filled with conductive metal particles are of particular interest for heat-dissipation applications in electronics packaging [1]. Another important application where increased thermal conductivity is required is in polymeric heat exchangers [2] when the thermal conductivity directly affects the performance of the exchanger. Low-cost metals may rapidly corrode in heat exchangers in aggressive environments or working fluids, and corrosionresistant polymeric materials have been considered as possible alternatives to metals in such devices. The higher thermal conductivity can be achieved by the use of suitable fillers such as aluminum, carbon fibers, graphite, aluminum nitrides, copper, etc. In fact, polymer composites may have thermal conductivities 10 to over 100 times higher than those of pure polymers, equal to or even higher than the thermal conductivities of some metals [3].

The effective thermal conductivity of a composite material has a complex dependence on the phases' properties, the size and shape of the filler particles, their distribution within the medium, filler-matrix interface characteristics, as well as on the temperature field itself. Various theories have been developed to model the thermal conductivity behavior in two-phase dispersed systems, and a number of secondorder models were found to provide good estimates for composites filled with low volumetric concentrations of particles, but with increasing deviations between real 
composite systems and theoretical models for high concentration values $[2,4,5]$. At such high concentrations, the filler particles tend to form agglomerates, and conductive chains may be formed that result in a marked increase on thermal conductivity [5]. A large number of contributions, for different combinations of filler and matrix material configurations, have provided comparisons of experimental and theoretical results [5-15] that confirm the lack of an universal model for thermal conductivity in two-phase dispersed systems.

As the composite material morphology in the realm of applications presents endless possibilities due to design tailoring, manufacturing processes and even selfstructuring, the characterization of their physical properties has to be made almost case to case. Accordingly, it becomes essential to develop a methodology for the identification of physical properties already on the finished material or system, via solution of the corresponding inverse problem for a space-variable thermal conductivity [16-19]. Thus, the problem that is addressed here is that of providing a reliable prediction of the composite thermal conductivity function from knowledge of the physical properties of each component in the mixture and their relative concentrations, without employing in advance a specific theoretical model among those proposed in the literature. Such inverse analysis would then allow for the verification of the adequacy of the existing models or even for their adaptation or formulation of novel predictive expressions.

The analysis of diffusion problems in heterogeneous media involves formulations with spatial variations of the thermophysical properties in different ways [20-23], such as due to variations on local concentrations in particle-filled composites and other two-phase dispersed systems. Regarding the solution of the direct heat conduction problem in the transient state, the procedure employed in this work stems from the application of the classical integral transform technique (CITT) $[24,25]$. Since we are dealing with a linear heat conduction problem with space-variable coefficients, integral transformation results into a decoupled transformed ordinary differential system, capable of being solved analytically. Nevertheless, the solution of the corresponding auxiliary eigenvalue problem for the classical integral transform technique demands the use of computational methodologies such as the sign-count method or the generalized integral transform technique (GITT) [25-28]. In this work we make use of the generalized integral transform technique, which has been successfully applied to the solution of eigenvalue problems with variable coefficients and irregular domains [25, 29-32]. Another possibility exploited here is to express the variable coefficients themselves as eigenfunction expansions [32]. This procedure is particularly useful for the fully analytical evaluation of the coefficients of the algebraic system in the transformed eigenvalue problem. Therefore, all manipulations can be expressed in terms of simpler eigenfunctions, allowing the analytical integrations and additional derivations to be performed in symbolic computation platforms [33].

Among the various available solution techniques for inverse problems [34-36], a fairly common approach is related to the minimization of an objective function that usually involves the quadratic difference between measured and estimated values, such as the least-squares norm, possibly with the inclusion of regularization terms. Despite the fact that the minimization of the least-squares norm is used indiscriminately, it yields only maximum-likelihood estimates if the following statistical 
hypotheses are valid: The errors in the measured variables are additive, uncorrelated, normally distributed, with zero mean, and known constant standard deviation; only the measured variables appearing in the objective function contain errors; and there is no prior information regarding the values and uncertainties of the unknown parameters [34].

Although very popular and useful in many situations, the minimization of the least-squares norm is a non-Bayesian estimator. A Bayesian estimator [36] is basically concerned with the analysis of the posterior probability density, which is the conditional probability of the parameters given the measurements, while the likelihood is the conditional probability of the measurements given the parameters. If we assume the parameters and the measurement errors to be independent Gaussian random variables, with known means and covariance matrices, and that the measurement errors are additive, a closed-form expression can be derived for the posterior probability density. In this case, the estimator that maximizes the posterior probability density can be recast in the form of a minimization problem involving the maximum a posteriori objective function. On the other hand, if different prior probability densities are assumed for the parameters, the posterior probability distribution may not allow an analytical treatment. Then, Markov chain Monte Carlo (MCMC) methods are used to draw samples of all possible parameters, so that inference on the posterior probability becomes inference on the samples.

This work illustrates the use of Bayesian inference in the estimation of spatially variable thermal conductivity in one-dimensional transient heat conduction problems of heterogeneous media, represented by two-phase dispersed systems, employing the method of Markov chain Monte Carlo [36-41]. The Metropolis-Hastings algorithm is used for the sampling procedure [42, 43], implemented in the Mathematica platform [33]. This sampling procedure is in general the most expensive computational task in solving an inverse problem by the method of Monte Carlo with Markov chain, since the direct problem is calculated for each state of the chain. In this context, the use of a fast, accurate, and robust computational implementation of the direct solution is extremely important. Thus, the integral transformation approach discussed above becomes very attractive for combined use with the Bayesian estimation procedure, since all steps in the method are determined analytically at once by symbolic computation and the single numerical repetitive task is the solution of an algebraic matrix eigenvalue problem [32].

Instead of seeking the function estimation in the form of local values for the thermal conductivity, an alternative approach is proposed here, which is based on the eigenfunction expansion of the thermal conductivity itself. Then the unknown parameters become the corresponding series coefficients.

Simulated temperatures obtained via integral transforms are used in the inverse analysis. From the prescription of the concentration distribution of the dispersed phase, available correlations for the thermal conductivity are employed to produce the simulated data with high precision in the direct problem solution, while eigenfunction expansions with reduced number of terms are employed in the inverse analysis itself, in order to avoid the so-called inverse crime [36]. Both Gaussian and noninformative uniform distributions were used as priors for comparison purposes. In addition, alternative correlations for the thermal conductivity that yield 
different predictions are also employed as Gaussian priors for the algorithm in order to test the inverse analysis robustness.

\section{DIRECT PROBLEM SOLUTION: INTEGRAL TRANSFORMS}

We consider the one-dimensional version of the general formulation on transient heat conduction presented and solved in [32], for the potential $T(x, t)$, dependent on position $x$ and time $t$ and defined in region $x \in[0, L]$. The formulation includes the transient term, the diffusion operator, a linear dissipation term, an independent heat-generation term, and the space-variable thermal conductivity and heat capacity, as shown in problem (1) below. The coefficients $w(x)$ and $k(x)$ are thus responsible for the information related to the heterogeneity of the medium. The diffusion equation and initial and boundary conditions are given by

$$
\begin{gathered}
w(x) \frac{\partial T(x, t)}{\partial t}=\frac{\partial}{\partial x}\left[k(x) \frac{\partial T(x, t)}{\partial x}\right]-d(x) T(x, t)+P(x, t) \quad x \in[0, L], t>0 \\
T(x, 0)=f(x) \quad x \in[0, L] \\
\alpha_{0} T(x, t)-\beta_{0} k(x) \frac{\partial T(x, t)}{\partial x}=\phi_{0}(t) \quad x=0, t>0 \\
\alpha_{1} T(x, t)+\beta_{1} k(x) \frac{\partial T(x, t)}{\partial x}=\phi_{1}(t) \quad x=L, t>0
\end{gathered}
$$

Problem (1) covers a fairly wide range of physical conditions for a typical one-dimensional transient thermal conductivity experimental setup, including the various types of boundary conditions and independent heating, or, for instance, based on an initial space-variable thermal excitation throughout the domain and subsequent temperature measurements acquisition via infrared thermography [40]. Before providing the integral transform solution of problem (1), a filtering solution is proposed which attempts to eliminate the source terms in the governing equation and boundary conditions, for improved convergence behavior of the eigenfunction expansions. In this case, we write the original temperature field as

$$
T(x, t)=T_{f}(x ; t)+T^{*}(x, t)
$$

The quasi-steady formulation of problem (1) may, for instance, be used to obtain the filter $T_{f}(x ; t)$, that is,

$$
\begin{array}{cl}
\frac{\partial}{\partial x}\left[k(x) \frac{\partial T_{f}(x ; t)}{\partial x}\right]-d(x) T_{f}(x ; t)+P(x, t)=0 & x \in[0, L] \\
\alpha_{0} T_{f}(x ; t)-\beta_{0} k(x) \frac{\partial T_{f}(x ; t)}{\partial x}=\phi_{0}(t) & x=0 \\
\alpha_{1} T_{f}(x ; t)+\beta_{1} k(x) \frac{\partial T_{f}(x ; t)}{\partial x}=\phi_{1}(t) \quad x=L
\end{array}
$$


where time $t$ enters the formulation as a parameter [26]. Simpler analytical filters may be preferred, which essentially homogenizes the original boundary conditions, Eqs. $(1 c, d)$, and eventually include simplified forms of the equation source term.

The filtered temperature formulation is then obtained as

$$
\begin{gathered}
w(x) \frac{\partial T^{*}(x, t)}{\partial t}=\frac{\partial}{\partial x}\left[k(x) \frac{\partial T^{*}(x, t)}{\partial x}\right]-d(x) T^{*}(x, t)+P^{*}(x, t) \quad x \in[0, L], t>0 \\
T^{*}(x, 0)=f^{*}(x) \quad x \in[0, L] \\
\alpha_{0} T^{*}(x, t)-\beta_{0} k(x) \frac{\partial T^{*}(x, t)}{\partial x}=0 \quad x=0, t>0 \\
\alpha_{1} T^{*}(x, t)+\beta_{1} k(x) \frac{\partial T^{*}(x, t)}{\partial x}=0 \quad x=L, t>0
\end{gathered}
$$

where the filtered initial condition and the filtered source term are given by

$$
P^{*}(x, t)=-w(x) \frac{\partial T_{f}(x ; t)}{\partial t} \quad f^{*}(x)=f(x)-T_{f}(x ; 0)
$$

The formal exact solution of problem (4) is then obtained with the classical integral transform method [24] and is written as

$$
T^{*}(x, t)=\sum_{i=1}^{\infty} \tilde{\psi}_{i}(x)\left[\bar{f}_{i} e^{-\mu_{i}^{2} t}+\int_{0}^{t} \bar{g}_{i}\left(t^{\prime}\right) e^{-\mu_{i}^{2}\left(t-t^{\prime}\right)} d t^{\prime}\right]
$$

where the eigenvalues $\mu_{i}$ and eigenfunctions $\psi_{i}(x)$ are obtained from the eigenvalue problem that contains the information about the heterogeneous medium in the form

$$
\frac{d}{d x}\left[k(x) \frac{d \psi_{i}(x)}{d x}\right]+\left(\mu_{i}^{2} w(x)-d(x)\right) \psi_{i}(x)=0 \quad x \in[0, L]
$$

with boundary conditions

$$
\begin{array}{ll}
\alpha_{0} \psi_{i}(x)-\beta_{0} k(x) \frac{d \psi_{i}(x)}{d x}=0 & x=0 \\
\alpha_{1} \psi_{i}(x)+\beta_{1} k(x) \frac{d \psi_{i}(x)}{d x}=0 & x=L
\end{array}
$$

Also, the other quantities that appear in the exact solution (6) are computed after solving problem (7), such as

$$
\begin{gathered}
N_{i}=\int_{0}^{L} w(x) \psi_{i}^{2}(x) d x \quad \text { normalization integrals } \\
\tilde{\psi}_{i}(x)=\frac{\psi_{i}(x)}{\sqrt{N_{i}}} \quad \text { normalized eigenfunctions }
\end{gathered}
$$




$$
\begin{array}{cc}
\bar{f}_{i}=\int_{0}^{L} w(x) \tilde{\Psi}_{i}(x) f^{*}(x) d x & \text { transformed initial condition } \\
\bar{g}_{i}(t)=\int_{0}^{L} P^{*}(x, t) \tilde{\Psi}_{i}(x) d x & \text { transformed source terms }
\end{array}
$$

For a general-purpose automatic implementation, it is quite desirable to develop a flexible computational approach to handle eigenvalue problems with arbitrarily variable coefficients, such as problem (7). Thus, the generalized integral transform technique is employed here in the solution of the Sturm-Liouville problem (7) via the proposition of a simpler auxiliary eigenvalue problem, and expanding the unknown eigenfunctions in terms of the chosen basis. Also, the variable equation coefficients are themselves expanded in terms of known eigenfunctions [32], so as to allow for a fully analytical implementation of the coefficient matrices in the transformed system. The solution of problem (7) is thus proposed as an eigenfunction expansion, in terms of a simpler auxiliary eigenvalue problem, given as

$$
\frac{d}{d x}\left[k^{*}(x) \frac{d \Omega_{n}(x)}{d x}\right]+\left[\lambda_{n}^{2} w^{*}(x)-d^{*}(x)\right] \Omega_{n}(x)=0 \quad x \in[0, L]
$$

with boundary conditions

$$
\begin{array}{ll}
\alpha_{0} \Omega_{n}(x)-\beta_{0} k^{*}(x) \frac{d \Omega_{n}(x)}{d x}=0 & x=0 \\
\alpha_{1} \Omega_{n}(x)+\beta_{1} k^{*}(x) \frac{d \Omega_{n}(x)}{d x}=0 & x=L
\end{array}
$$

where the coefficients $w^{*}(x), k^{*}(x)$, and $d^{*}(x)$ are simpler forms of the equation coefficients, chosen to allow for an analytical solution of the auxiliary problem.

The proposed expansion of the original eigenfunction is then given by

$$
\begin{gathered}
\psi_{i}(x)=\sum_{n=1}^{\infty} \widetilde{\Omega}_{n}(x) \bar{\psi}_{i, n} \quad \text { inverse } \\
\bar{\psi}_{i, n}=\int_{0}^{L} w^{*}(x) \psi_{i}(x) \widetilde{\Omega}_{n}(x) d x \quad \text { transform }
\end{gathered}
$$

where the normalization integral and normalized auxiliary eigenfunction are given by

$$
\begin{aligned}
M_{n} & =\int_{0}^{L} w^{*}(x) \widetilde{\Omega}_{n}^{2}(x) d x \quad \text { norm } \\
\widetilde{\Omega}_{n}(x) & =\frac{\Omega_{n}(x)}{\sqrt{M_{n}}} \quad \text { normalized eigenfunction }
\end{aligned}
$$


The integral transformation is thus performed by operating on Eq. (7a) with $\int_{0}^{L} \widetilde{\Omega}_{n}(x)-d x$ to yield, after some manipulation [32], the following algebraic problem in matrix form:

$$
\begin{gathered}
(\mathbf{A}-\nu \mathbf{B}) \overline{\boldsymbol{\Psi}}=0 \quad \text { with } \nu=\mu^{2} \\
\overline{\boldsymbol{\Psi}}=\left\{\overline{\boldsymbol{\psi}}_{n}^{(m)}\right\} \quad \mathbf{B}=\left\{B_{n, m}\right\} \quad B_{n, m}=\int_{0}^{L} w(x) \widetilde{\boldsymbol{\Omega}}_{n}(x) \widetilde{\boldsymbol{\Omega}}_{m}(x) d x \quad(11 b-d) \\
A_{n, m}=-\int_{0}^{L} \widetilde{\boldsymbol{\Omega}}_{m}(x) \frac{d}{d x}\left[k(x) \frac{d \widetilde{\boldsymbol{\Omega}}_{n}(x)}{d x}\right] d x+\left\{\left[\left(k(x)-k^{*}(x)\right]\left[\widetilde{\boldsymbol{\Omega}}_{m}(x) \frac{d \widetilde{\boldsymbol{\Omega}}_{n}(x)}{d x}\right]\right\}_{x=0}^{x=L}\right. \\
+\int_{0}^{L} d(x) \widetilde{\boldsymbol{\Omega}}_{n}(x) \widetilde{\boldsymbol{\Omega}}_{m}(x) d x \\
A=\left\{A_{n, m}\right\}
\end{gathered}
$$

The algebraic problem (11) can be solved numerically to provide results for the eigenvalues and eigenvectors, upon truncation to a sufficiently large finite order $M$, which will be combined by the inverse formula $(10 a)$ to provide the desired original eigenfunctions.

As mentioned previously, it is also relevant to consider the possibility of expressing the variable coefficients themselves as eigenfunction expansions [32]. This is particularly advantageous in the evaluation of the algebraic system coefficients, $A_{n, m}$ and $B_{n, m}$. All the related integrals can then be expressed in terms of eigenfunctions, allowing for straightforward analytical evaluations. For instance, the coefficient $w(x)$ can be expanded in terms of eigenfunctions, together with a filtering solution to enhance convergence, in the following form:

$$
\begin{array}{cc}
w(x)=w_{f}(x)+\sum_{k=1}^{\infty} \widetilde{\Gamma}_{k}(x) \bar{w}_{k} \quad \text { inverse } \\
\bar{w}_{k}=\int_{0}^{L} \hat{w}(x)\left[w(x)-w_{f}(x)\right] \widetilde{\Gamma}_{k}(x) d x & \text { transform }
\end{array}
$$

where $\hat{w}(x)$ is the weighting function for the chosen normalized eigenfunction $\widetilde{\Gamma}_{k}(x)$. For instance, the eigenfunction basis may be chosen employing the same auxiliary problem equation, but with first-order boundary conditions throughout, while the filtering function would be a simple analytic function that satisfies the boundary values for the original coefficients. Then, once the transformed coefficients have been obtained through the transform formula, Eq. (12b), computations may be carried on with the inverse expression for the variable coefficient, Eq. (12a). The two remaining coefficients are equally expanded in terms of eigenfunctions to yield

$$
\begin{gathered}
k(x)=k_{f}(x)+\sum_{k=1}^{\infty} \widetilde{\Gamma}_{k}(x) \bar{k}_{k} \quad \text { inverse } \\
\bar{k}_{k}=\int_{0}^{L} \hat{w}(x)\left[k(x)-k_{f}(x)\right] \widetilde{\Gamma}_{k}(x) d x \quad \text { transform }
\end{gathered}
$$




$$
\begin{aligned}
& d(x)=d_{f}(x)+\sum_{k=1}^{\infty} \widetilde{\Gamma}_{k}(x) \bar{d}_{k} \quad \text { inverse } \\
& \bar{d}_{k}=\int_{0}^{L} \hat{w}(x)\left[d(x)-d_{f}(x)\right] \widetilde{\Gamma}_{k}(x) d x \quad \text { transform }
\end{aligned}
$$

The matrices coefficients may then be rewritten in terms of the expanded functions, such as for the elements of matrix $\mathbf{B}$,

$$
B_{n, m}=\int_{0}^{L} w_{f}(x) \widetilde{\boldsymbol{\Omega}}_{n}(x) \widetilde{\boldsymbol{\Omega}}_{m}(x) d x+\sum_{k=1}^{\infty} \bar{w}_{k} \int_{0}^{L} \widetilde{\boldsymbol{\Gamma}}_{k}(x) \widetilde{\boldsymbol{\Omega}}_{n}(x) \widetilde{\boldsymbol{\Omega}}_{m}(x) d x
$$

and for matrix A,

$$
\begin{aligned}
A_{n, m}= & -\int_{0}^{L} \widetilde{\boldsymbol{\Omega}}_{m}(x) \frac{d}{d x}\left[k_{f}(x) \frac{d \widetilde{\boldsymbol{\Omega}}_{n}(x)}{d x}\right] d x-\sum_{k=1}^{\infty}\left\{\int_{0}^{L} \widetilde{\boldsymbol{\Omega}}_{m}(x) \frac{d}{d x}\left[\widetilde{\boldsymbol{\Gamma}}_{k}(x) \frac{d \widetilde{\boldsymbol{\Omega}}_{n}(x)}{d x}\right] d x\right\} \overline{\boldsymbol{k}}_{k} \\
& +\left\{\left[k_{f}(x)-k^{*}(x)\right]\left[\widetilde{\boldsymbol{\Omega}}_{m}(x) \frac{d \widetilde{\boldsymbol{\Omega}}_{n}(x)}{d x}\right]\right\}_{x=0}^{x=L}+\sum_{k=1}^{\infty}\left[\left\{\left[\widetilde{\boldsymbol{\Gamma}}_{k}(x)\right]\left[\widetilde{\boldsymbol{\Omega}}_{m}(x) \frac{d \widetilde{\boldsymbol{\Omega}}_{n}(x)}{d x}\right]\right\}_{x=0}^{x=L} \bar{k}_{k}\right. \\
& +\int_{0}^{L} d_{f}(x) \widetilde{\boldsymbol{\Omega}}_{n}(x) \widetilde{\boldsymbol{\Omega}}_{m}(x) d x+\sum_{k=1}^{\infty}\left[\int_{0}^{L} \widetilde{\boldsymbol{\Gamma}}_{k}(x) \widetilde{\boldsymbol{\Omega}}_{n}(x) \widetilde{\boldsymbol{\Omega}}_{m}(x) d x\right] \bar{d}_{k}
\end{aligned}
$$

Also, the normalization integrals are then computed from

$$
N_{i}=\sum_{n=1}^{\infty} \sum_{m=1}^{\infty} \bar{\psi}_{i, n} \bar{\psi}_{i, m}\left\{\int_{0}^{L} w_{f}(x) \widetilde{\boldsymbol{\Omega}}_{n}(x) \widetilde{\boldsymbol{\Omega}}_{m}(x) d x+\sum_{k=1}^{\infty}\left[\int_{0}^{L} \widetilde{\boldsymbol{\Gamma}}_{k}(x) \widetilde{\boldsymbol{\Omega}}_{n}(x) \widetilde{\boldsymbol{\Omega}}_{m}(x) d x\right] \overline{\boldsymbol{w}}_{k}\right\}
$$

This procedure will also be of interest in the function estimation task, when the transformed coefficients will be the parameters to be estimated.

\section{INVERSE PROBLEM SOLUTION: BAYESIAN INFERENCE}

Consider the vector of parameters appearing in the physical model formulation as

$$
\mathbf{P}^{T} \equiv\left[P_{1}, P_{2}, \ldots, P_{N p}\right]
$$

where $N_{p}$ is the number of parameters. For the solution of the inverse problem of estimating $\mathbf{P}$, we assume available the measured temperature data given by

$$
(\mathbf{Y}-\mathbf{T})^{T}=\left(\vec{Y}_{1}-\vec{T}_{1}, \vec{Y}_{2}-\vec{T}_{2}, \ldots, \vec{Y}_{N_{x}}-\vec{T}_{N_{x}}\right)
$$


where $\mathbf{T}$ is the vector of estimated temperatures and $\vec{Y}_{i}$ contains the measured temperatures for each of the $N_{x}$ sensors at time $t_{i}, i=1, \ldots, N_{t}$, that is,

$$
\left(\vec{Y}_{i}-\vec{T}_{i}\right)=\left(Y_{i 1}-T_{i 1}, Y_{i 2}-T_{i 2}, \ldots, Y_{i N_{x}}-T_{i N_{x}}\right) \quad \text { for } i=1, \ldots, N_{t}
$$

so that we have $N_{m}=N_{x}$. $N_{t}$ measurements in total.

Bayes's theorem can then be stated as [36-39]

$$
p_{\text {posterior }}(\mathbf{P})=p(\mathbf{P} \mid \mathbf{Y})=\frac{p(\mathbf{P}) p(\mathbf{Y} \mid \mathbf{P})}{p(\mathbf{Y})}
$$

where $p_{\text {posterior }}(\mathbf{P})$ is the posterior probability density, that is, the conditional probability of the parameters $\mathbf{P}$ given the measurements $\mathbf{Y} ; p(\mathbf{P})$ is the prior density, that is, a statistical model for the information about the unknown parameters prior to the measurements; $p(\mathbf{Y} \mid \mathbf{P})$ is the likelihood function, which gives the relative probability density (loosely speaking, relative probability) of different measurement outcomes $\mathbf{Y}$ with a fixed $\mathbf{P}$; and $p(\mathbf{Y})$ is the marginal probability density of the measurements, which plays the role of a normalizing constant.

In this work we assume that the measurement errors are Gaussian random variables, with known (modeled) means and covariances, and that the measurement errors are additive and independent of the unknowns. With these hypotheses, the likelihood function can be expressed as [34-39]

$$
p(\mathbf{Y} \mid \mathbf{P})=(2 \pi)^{-N_{m} / 2}|\mathbf{W}|^{-1 / 2} \exp \left\{-\frac{1}{2}[\mathbf{Y}-\mathbf{T}(\mathbf{P})]^{T} \mathbf{W}^{-1}[\mathbf{Y}-\mathbf{T}(\mathbf{P})]\right\}
$$

where $\mathbf{W}$ is the covariance matrix of the measurement errors.

When it is not possible to obtain the corresponding marginal distributions analytically, one needs to use a method based on simulation [36, 38]. The inference based on simulation techniques uses samples to extract information about the posterior distribution $\boldsymbol{p}(\mathbf{P} \mid \mathbf{Y})$. Obviously, as a sample is always a partial substitute of the information contained in a density, simulation-based methods are inherently approximate and should only be used when it is impossible to include the extraction of analytical information from the posteriori, as is the case in the present study. Unfortunately, for most problems of practical relevance it is complicated to generate the posteriori $\boldsymbol{p}(\mathbf{P} \mid \mathbf{Y})$. Therefore, more sophisticated methods are required to obtain a sample of $\boldsymbol{p}(\mathbf{P} \mid \mathbf{Y})$, for example, the simulation technique based on Markov chains [38]. The numerical method most used to explore the space of states of the posteriori is the Monte Carlo approach. The Monte Carlo simulation is based on a large number of samples of the probability density function [in this case, the function of the posterior probability density $\boldsymbol{p}(\mathbf{P} \mid \mathbf{Y})$ ]. Several sampling strategies are proposed in the literature, including the Monte Carlo method with Markov chain, adopted in this work, where the basic idea is to simulate a "random walk" in the space of $p(\mathbf{P} \mid \mathbf{Y})$ that converges to a stationary distribution, which is the distribution of interest in the problem.

A Markov chain is a stochastic process $\left\{\mathbf{P}_{0}, \mathbf{P}_{1}, \ldots\right\}$ such that the distribution of $\mathbf{P}_{i}$, given all previous values $\mathbf{P}_{0}, \ldots, \mathbf{P}_{i-1}$, depends only on $\mathbf{P}_{i-1}$. That is, it interprets the fact that for a process satisfying the Markov property of Eq. (17), given the 
present, the past is irrelevant to predict its position in a future instant [38]:

$$
p\left(\mathbf{P}_{i} \in A \mid \mathbf{P}_{0}, \ldots, \mathbf{P}_{i-1}\right)=p\left(\mathbf{P}_{i} \in A \mid \mathbf{P}_{i-1}\right)
$$

The most commonly used Monte Carlo method with Markov chain algorithms are the Metropolis-Hastings, employed here, and the Gibbs sampler [38, 39].

The Markov chain according to the generic label of Metropolis-Hastings comes from the articles of Metropolis et al. [42] and Hastings [43], later on complemented by the works of Barker [44] and Peskun [45]. The Metropolis-Hastings algorithm uses the same idea of the rejection methods, i.e., a value is generated from an auxiliary distribution and accepted with a given probability. This correction mechanism ensures the convergence of the chain for the equilibrium distribution. That is, the algorithm now includes an additional step, where the transition mechanism depends on a proposal for a transition and a stage of assessing the equilibrium density, but this is represented by the global transition via the probability of acceptance.

The Metropolis-Hastings algorithm uses an auxiliary probability density function, $q\left(\mathbf{P}^{*} \mid \mathbf{P}\right)$, from which it is easy to obtain sample values. Assuming that the chain is in a state $\mathbf{P}$, a new candidate value, $\mathbf{P}^{*}$, is generated from the auxiliary distribution $q\left(\mathbf{P}^{*} \mid \mathbf{P}\right)$, given the current state of the chain $\mathbf{P}$.

The new value $\mathbf{P}^{*}$ is accepted with probability given by Eq. (18), where the ratio that appears in this equation was called by Hastings [43] the ratio test, and is today called the ratio of Hastings " $\mathrm{RH}$ ":

$$
\operatorname{RH}\left(\mathbf{P}, \mathbf{P}^{*}\right)=\min \left[1, \frac{p\left(\mathbf{P}^{*} \mid \mathbf{Y}\right) q\left(\mathbf{P}^{*} \mid \mathbf{P}\right)}{p(\mathbf{P} \mid \mathbf{Y}) q\left(\mathbf{P} \mid \mathbf{P}^{*}\right)}\right]
$$

where $p(\mathbf{P} \mid \mathbf{Y})$ is the a posteriori distribution of interest. An important observation is that we only need to know $p(\mathbf{P} \mid \mathbf{Y})$ up to a constant, since we are working with ratios between densities and such normalization constant is canceled.

In practical terms, this means that the simulation of a sample of $p(\mathbf{P} \mid \mathbf{Y})$ using the Metropolis-Hastings algorithm can be outlined as follows [38]:

1. Boot up the iterations counter of the chain $i=0$ and assign an initial value $\mathbf{P}^{(0)}$.

2. Generate a candidate value $\mathbf{P}^{*}$ of the distribution $q\left(\mathbf{P}^{*} \mid \mathbf{P}\right)$.

3. Calculate the probability of acceptance of the candidate value $\mathrm{RH}\left(\mathbf{P}, \mathbf{P}^{*}\right)$ by Eq. (18).

4. Generate a random number $u$ with uniform distribution, i.e., $u \sim \mathrm{U}(0,1)$.

5. If $u \leq \mathrm{RH}$, then the new value is accepted and we let $\mathbf{P}^{(i+1)}=\mathbf{P}^{*}$. Otherwise, the new value is rejected and we let $\mathbf{P}^{(i+1)}=\mathbf{P}^{(i)}$.

6. Increase the counter $i$ to $i+1$ and return to step 2 .

The transition core $q\left(\mathbf{P}^{*} \mid \mathbf{P}\right)$ defines only a proposal for a movement that can be confirmed by $\operatorname{RH}\left(\mathbf{P}, \mathbf{P}^{*}\right)$. For this reason it is usually called the proposal or density distribution. The success of the method depends on not so low acceptance rates and proposals that are easy to simulate. The method replaces a difficult-to-generate $p(\mathbf{P} \mid \mathbf{Y})$ by several generations of the proposal $q\left(\mathbf{P}^{*} \mid \mathbf{P}\right)$. In this study we have chosen to adopt symmetrical chains, i.e., $q\left(\mathbf{P}^{*} \mid \mathbf{P}\right)=q\left(\mathbf{P} \mid \mathbf{P}^{*}\right)$ for all $\left(\mathbf{P}^{*}, \mathbf{P}\right)$. In this case, 
Eq. (18) reduces to the ratio of the posterior densities calculated at the previous and proposed chain positions, and does not depend on $q\left(\mathbf{P}^{*} \mid \mathbf{P}\right)$.

The unknown quantity in the heat conduction problem addressed here is the thermal conductivity of the medium, $k(x)$. However, the approach adopted in solving the direct problem was to expand the thermal conductivity function in terms of eigenfunctions, so that the unknown quantities are in fact the coefficients of the eigenfunction expansion and the two values of the property at the boundaries, employed in the solution procedure as a filter.

\section{RESULTS AND DISCUSSION}

The inverse problem solution illustrated here involves the analysis of an abrupt variation of the thermal conductivity in a two-phase dispersed system. In order to examine the accuracy and robustness of the proposed inverse analysis, we have made use of simulated measured temperature data along the length of the domain, in the transient regime, such as obtained through infrared thermography [41]. Such measurements were obtained from the solution of the direct (forward) problem by specifying the functions and values for the filler concentration distribution and thermophysical properties. The simulated data were disturbed by an error with a mean value centered on the exact temperature and constant and known variance, in order to simulate actual measured temperature data. For the results of the inverse analysis to be presented below, we have employed the parameter values shown in Table 1 for the generation of the simulated measured data, as extracted from [9] for a polyethylene matrix filled with alumina particles.

The dimensionless problem formulation in this case reduces to

$$
w(x) \frac{\partial T(x, t)}{\partial t}=\frac{\partial}{\partial x}\left[k(x) \frac{\partial T(x, t)}{\partial x}\right] \quad 0<x<1, \quad t>0
$$

with initial and boundary conditions

$$
T(x, 0)=f(x) \quad 0<x<1
$$

Table 1. Parameter values used to generate the simulated measurement data [9]

Dimensionless length

Percent filler concentration at $x=0$

Percent filler concentration at $x=L$

Matrix properties (HDPE)

Filler properties (alumina)

Effective thermal conductivity model Parameters in filler concentration function

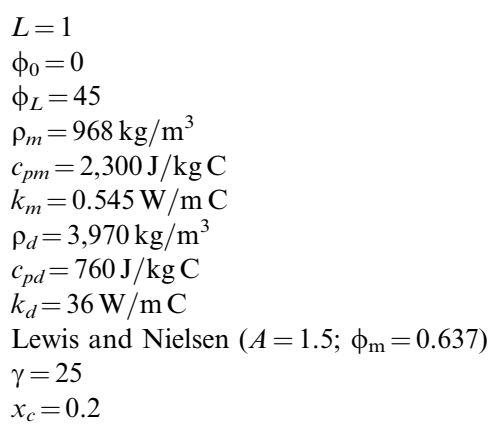




$$
\left.\frac{\partial T(x, t)}{\partial x}\right|_{x=0}=\left.0 \quad \frac{\partial T(x, t)}{\partial x}\right|_{x=1}=0 \quad t>0
$$

For the present illustration the initial condition was chosen arbitrarily from a random-number generation between 0 and 1, shown in Figure 1, as obtained from an initial flash lamp heating with a randomly perforated screen [41]. The space-variable distribution for the abrupt variation of the concentration of the filler into the matrix is governed by the parameter $\gamma$ in the function below:

$$
\begin{gathered}
\phi(x)=\phi_{x=0}+\left(\phi_{x=L}-\phi_{x=0}\right) \delta(x) \\
\delta(x)=\frac{1}{1+e^{-\gamma\left(x-x_{c}\right)}}
\end{gathered}
$$

with $x_{c}$ being the transition position between the regions of lower and higher concentrations of the filler.

The direct problem solution was obtained from the generalized integral transform technique as described above. A thorough convergence analysis was performed to select the number of terms in the temperature expansion, as well as the number of terms in the coefficients expansion.

The eigenvalue problem that has been solved is then given by

$$
\frac{d}{d x}\left[k(x) \frac{d \psi_{i}(x)}{d x}\right]+\mu_{i}^{2} w(x) \psi_{i}(x)=0 \quad 0<x<1
$$

with boundary conditions

$$
\left.\frac{d \psi_{i}(x)}{d x}\right|_{x=0}=\left.0 \quad \frac{d \psi_{i}(x)}{d x}\right|_{x=1}=0
$$

So as to demonstrate the potential applicability of the present approach, the simplest possible auxiliary problem was considered, based on the choice of

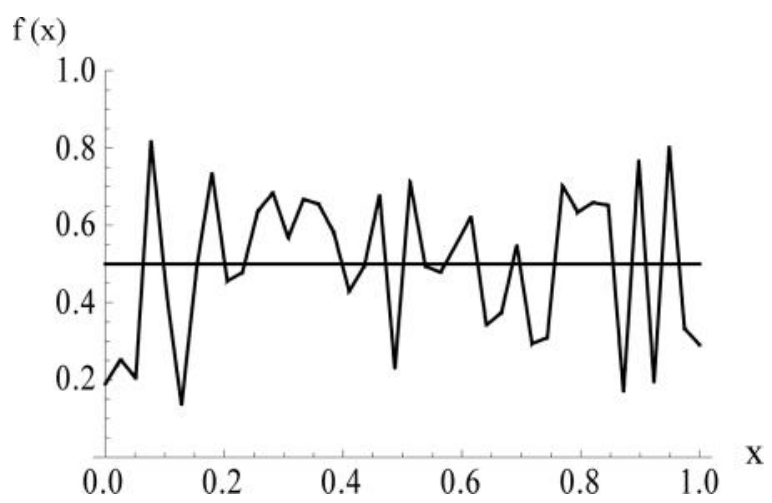

Figure 1. Initial dimensionless temperature distribution, randomly generated, employed in the direct problem solution. 
coefficients $k^{*}(x)=1, w^{*}(x)=1$, and $d^{*}(x)=0$, and maintaining the same boundary conditions as in Eqs. $(21 b, c)$, which results in:

$$
\widetilde{\Omega}_{n}(x)=\sqrt{2} \cos \left(\lambda_{n} x\right) \quad \text { and } \quad \widetilde{\Omega}_{0}(x)=1 \quad \text { with } \lambda_{n}=n \pi, n=0,1,2, \ldots
$$

From the availability of the filler concentration distribution along the domain, Eq. (20a), as obtainable from different possible techniques, the thermal capacity along the space coordinate is deterministically determined from mixtures theory. Thus, the coefficient $w(x)$ is considered as known in the inverse problem analysis, given as

$$
w(x)=1+\left(\frac{\rho_{d} c_{p d}}{\rho_{m} c_{p m}}-1\right) \phi(x)
$$

Figures $2 a$ and $2 b$ illustrate the behavior of the filler concentration distribution employed in the simulations that follow, besides the corresponding behavior of the dimensionless thermal capacity, according to Eq. (23) above, using $\gamma=25$ and $x_{c}=0.2$.

However, for the thermal conductivity determination, the volumetric content of the filler is not sufficiently informative to yield a good prediction of this physical property following the available theoretical approaches [9], especially for the higher concentration values. Many theoretical and empirical models have been proposed to predict the effective thermal conductivity of two-phase dispersed systems, and comprehensive review articles have discussed the applicability of many of these models [14]. Here, we discuss just a few of them that are relevant to the inverse analysis that follows. As upper and lower bounds of the effective thermal conductivity, we may point out the parallel or series arrangements with respect to heat flow, which yield the relations below, respectively:

$$
\begin{array}{rlr}
k_{c} & =(1-\phi) k_{m}+\phi k_{d} & \text { parallel conduction model } \\
k_{c}^{-1} & =(1-\phi) k_{m}^{-1}+\phi k_{d}^{-1} & \text { series conduction model }
\end{array}
$$

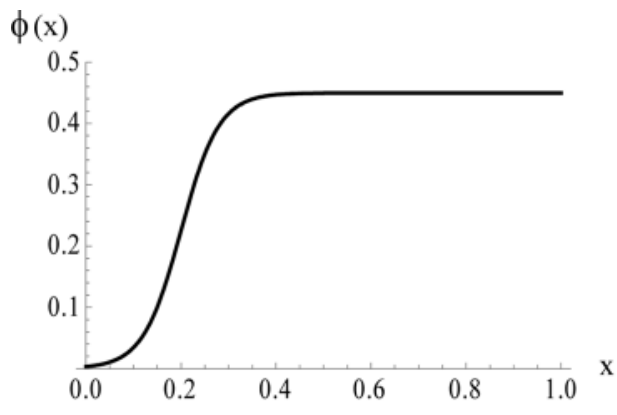

(a)

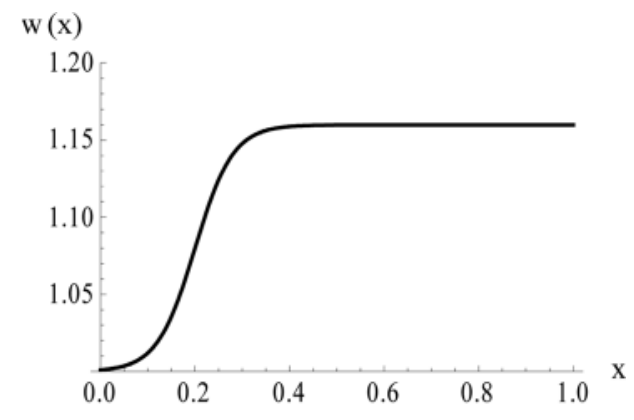

(b)

Figure 2. Sample behavior of the filler concentration distribution (a) and of the resulting dimensionless thermal capacity (b), according to the data in Table 1. 
A simple geometric mean model for the effective thermal conductivity of composites has also been employed in the literature [14]:

$$
k_{c}=k_{d}^{\phi} k_{m}^{(1-\phi)}
$$

One of the best-known models is that due to Maxwell [46], based on potential theory, in the form of an exact solution for the conductivity of randomly distributed and noninteracting homogeneous spheres in a homogeneous matrix:

$$
k_{c}=k_{m}\left[\frac{k_{d}+2 k_{m}+2 \phi\left(k_{d}-k_{m}\right)}{k_{d}+2 k_{m}-\phi\left(k_{d}-k_{m}\right)}\right]
$$

Maxwell's model predicts the effective thermal conductivities fairly well at low filler concentrations, but at higher concentrations the particles may form conductive chains, and the model underestimates the conductivity in such regions.

Lewis and Nielsen [47] proposed a model that attempts to include the effect of the shape of the particles and the orientation or type of packing for a two-phase system. The resulting expression is given as

$$
k_{c}=k_{m}\left(\frac{1+A B \phi}{1-B \phi \psi}\right) \quad \text { where } B=\frac{\left(k_{d} / k_{m}\right)-1}{\left(k_{d} / k_{m}\right)+A} \text { and } \psi=1+\left(\frac{1-\phi_{m}}{\phi_{m}^{2}}\right) \phi
$$

The values of $A$ and $\phi_{m}$ are suggested in [47] for a number of different geometric shapes and orientations, such as $A=1.50$ for spheres and $\phi_{m}=0.637$ for random packing.

Agari and Uno [48] proposed another model that attempts to combine the parallel and series conduction mechanisms, in the form

$$
\log k_{c}=\phi C_{2} \log k_{f}+(1-\phi) \log \left(C_{1} k_{m}\right)
$$

where $C_{1}$ and $C_{2}$ are experimentally determined constants of order unity. Since this model provides two parameters for empirical adjustment, in general it results in a better agreement with available experimental data. Figures $3 a-3 c$ illustrate the behavior of some of the above models for the present situation, according to the data in Table 1. Figure $3 a$ presents the curves corresponding to the two upper and lower bounds, the parallel and series models, respectively, as well as the geometric mean model, and the two models based on theoretical derivations. These two models by Maxwell and Lewis-Nielsen are more closely compared in Figure $3 b$. The percentage deviation between these last two models is presented in Figure $3 c$.

The nonparametric estimations in the present work were addressed to the coefficients of the eigenfunction expansion of $k(x)$ and the two values at the boundaries used in the linear filter function used in the expansion process. Thus, the parameters and the number of parameters to be estimated are given by

$$
\mathbf{P}=\left[k_{x=0}, k_{x=L}, \bar{k}_{1}, \bar{k}_{2}, \bar{k}_{3}, \ldots, \bar{k}_{N_{k}}\right] \quad \text { with } N_{P}=N_{k}+2
$$




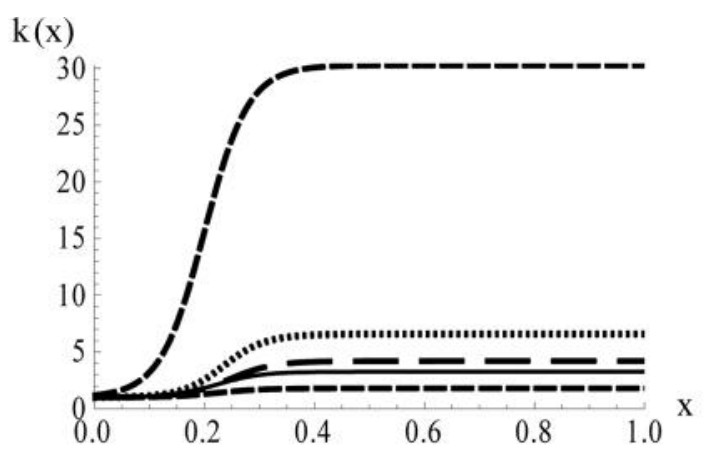

(a) Parallel model (uppermost dashed line), geometric mean (dots), Lewis \& Nielsen (long dashes), Maxwell (solid), series model (lowermost dashed line)

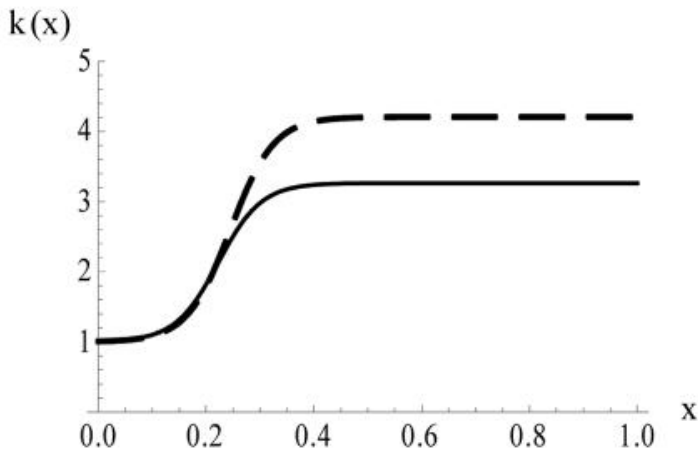

(b) Lewis \& Nielsen (dashed) and Maxwell (solid)

$\epsilon \%$

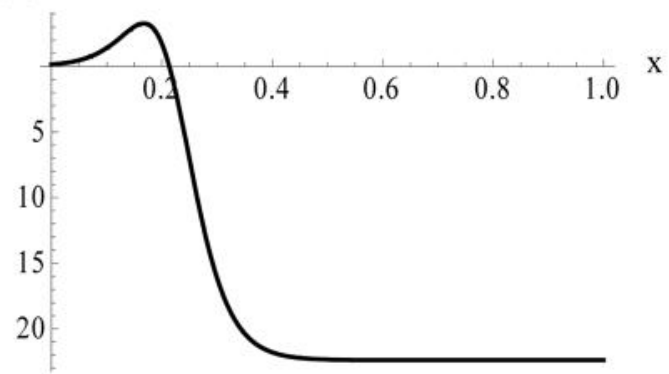

(c) Percentual deviation between Lewis \& Nielsen and Maxwell models.

Figure 3. Behavior of thermal conductivity models in proposed test case.

where

$$
k(x)=k_{f}(x)+\sum_{k=1}^{N_{k}} \widetilde{\Gamma}_{k}(x) \bar{k}_{k}
$$

In the proposed inverse approach, the truncation order of the thermal conductivity expansion, $N_{k}$, thus controls the number of parameters to be estimated. 
The convergence analysis of the $k(x)$ expansion, as obtained by the Lewis and Nielsen model, Eqs. (26), is shown in Figures $4 a-c$, for three different truncation orders, $N_{k}=4,7$, and 10 . It can be observed that the three increasing truncation orders given in these figures are able to recover the characteristic behavior of the chosen thermal conductivity function following the abrupt change in filler concentration, but the results for the lowest truncation order, $N_{k}=4$, still show some oscillation around the exact function, while for $N_{k}=10$ a much closer agreement between the expanded and the exact functions is observed.

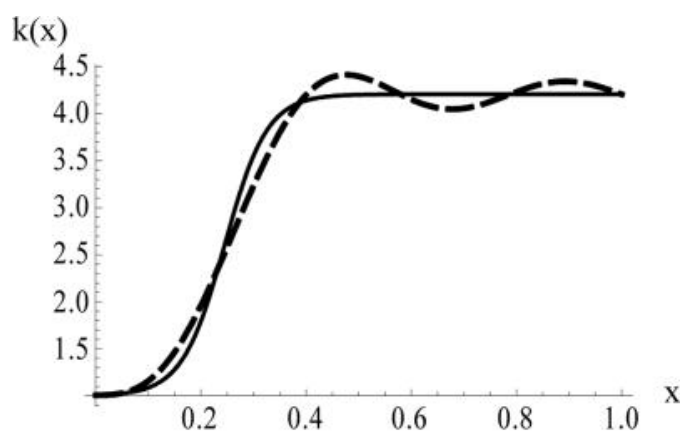

(a)

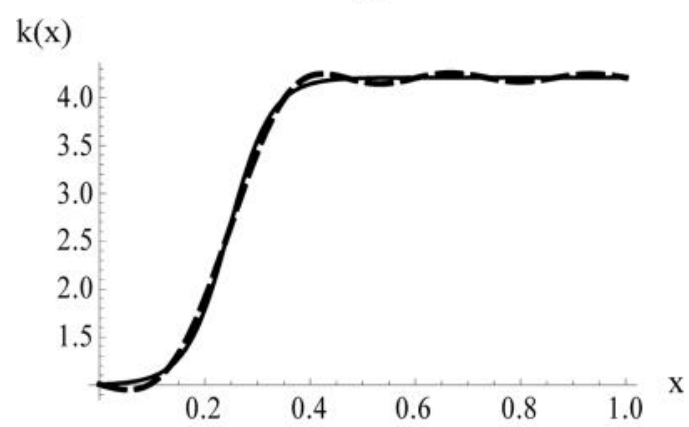

(b)

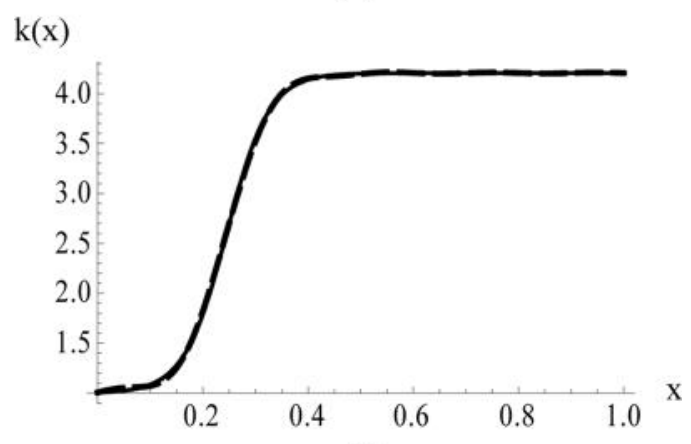

(c)

Figure 4. Convergence behavior of the thermal conductivity function-exact (solid line) and expansion (dashed line): (a) $N_{k}=4 ;(b) N_{k}=7$; and (c) $N_{k}=10$. 
Before addressing the estimation of the unknown parameters, the behavior of the determinant of the matrix $\mathbf{J}^{T} \mathbf{J}[34,35]$ was analyzed in order to inspect the influence of the number of parameters to be estimated in the solution of the inverse problem. The sensitivity matrix $\mathbf{J}$ is defined as:

$$
\mathbf{J}(\mathbf{P})=\left[\frac{\partial \mathbf{T}^{T}(\mathbf{P})}{\partial \mathbf{P}}\right]^{T}=\left[\begin{array}{ccccc}
\frac{\partial \vec{T}_{1}^{T}}{\partial P_{1}} & \frac{\partial \vec{T}_{1}^{T}}{\partial P_{2}} & \frac{\partial \vec{T}_{1}^{T}}{\partial P_{3}} & \cdots & \frac{\partial \vec{T}_{1}^{T}}{\partial P_{N p}} \\
\frac{\partial \vec{T}_{2}^{T}}{\partial P_{1}} & \frac{\partial \vec{T}_{2}^{T}}{\partial P_{2}} & \frac{\partial \vec{T}_{2}^{T}}{\partial P_{3}} & \cdots & \frac{\partial \vec{T}_{2}^{T}}{\partial P_{N_{p}}} \\
\vdots & \vdots & \vdots & & \vdots \\
\frac{\partial \vec{T}_{N_{t}}^{T}}{\partial P_{1}} & \frac{\partial \vec{T}_{N_{t}}^{T}}{\partial P_{2}} & \frac{\partial \vec{T}_{N_{t}}^{T}}{\partial P_{3}} & \cdots & \frac{\partial \vec{T}_{N_{t}}^{T}}{\partial P_{N_{p}}}
\end{array}\right]
$$

where

$$
\frac{\partial \vec{T}_{i}^{T}}{\partial P_{j}}=\left[\begin{array}{c}
\frac{\partial T_{i 1}^{T}}{\partial P_{j}} \\
\frac{\partial T_{i 2}^{T}}{\partial P_{j}} \\
\vdots \\
\frac{\partial T_{i N_{x}}^{T}}{\partial P_{j}}
\end{array}\right] \quad j=1,2, \ldots, N_{t}
$$

The sensitivity coefficients $J_{k j}=\partial T_{k} / \partial P_{j}, k=1,2, \ldots, N_{m}$ and $j=1,2, \ldots, N_{P}$, give the sensitivity of $T_{k}$ (solution of the direct problem) with respect to changes in the parameter $P_{j}$. A small value of the magnitude of $J_{k j}$ indicates that large changes in $P_{j}$ yield small changes in $T_{k}$. It can be easily noticed that the estimation of the parameter $P_{j}$ is extremely difficult in such cases, because basically the same value for $T_{k}$ would be obtained for a wide range of values of $P_{j}$. In fact, when the sensitivity coefficients are small, $\left|\mathbf{J}^{T} \mathbf{J}\right| \approx 0$, the inverse problem is said to be ill-conditioned. It can also be shown that $\left|\mathbf{J}^{T} \mathbf{J}\right|$ is null if any column of $\mathbf{J}$ can be expressed as a linear combination of other columns [34]. Therefore, it is desirable to have linearly independent sensitivity coefficients $J_{k j}$ with large magnitudes, so that the parameter estimation problem is not very sensitive to measurement errors and accurate estimates of the parameters can be obtained. The comparison of the magnitude of the sensitivity coefficients, as well as the analysis of possible linear dependence, is more easily performed by using the reduced sensitivity coefficients instead of the original ones. The reduced sensitivity coefficients are obtained by multiplying the original sensitivity coefficients, $J_{k j}$, by the parameters to which they refer to. Therefore, they have units of the measured variables, which are used as a basis of comparison.

Based on possible experimental setups, we will consider the following two cases for the analysis of the determinant of the information matrix $\mathbf{J}^{T} \mathbf{J}$ : (1) variation of the number of parameters to be estimated with a fixed number of spatial measurements and a fixed frequency of measurements (Figure $5 a$ ); (2) variation of the number of spatial measurements with a fixed frequency of measurements and a fixed number of parameters (Figure 5b). 


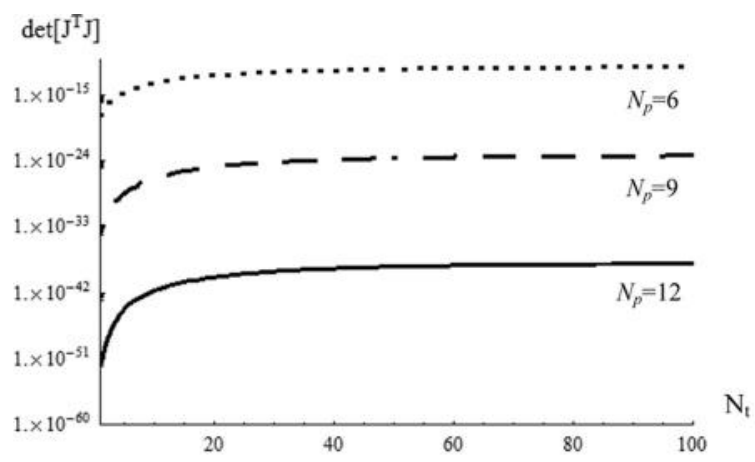

(a) $N_{x}=200, \Delta t=0.0005,\left(N_{P}=6,9\right.$ and 12 parameters $)$

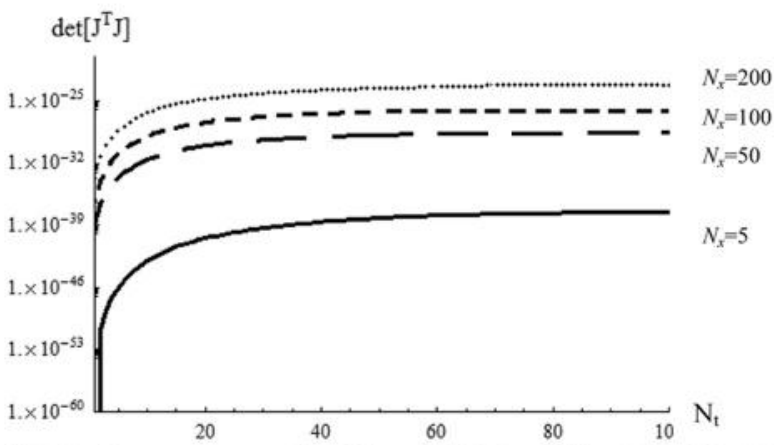

(b) $N_{P}=9$ parameters, fixed $\Delta t=0.0005$ and $N_{x}=200,100,50$, and 5 .

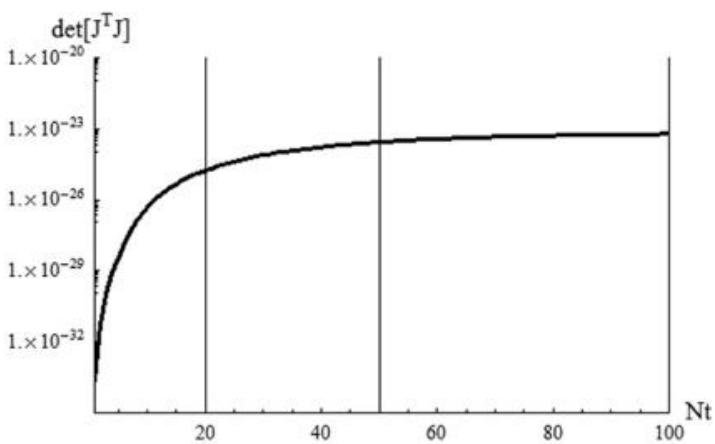

(c) $N_{P}=9$ parameters, $N_{x}=200, \Delta t=0.0005$ and $N_{f}=20,50$ and 100

Figure 5. Evolution of sensitivity matrix determinant.

Figure $5 a$ shows the evolution in time of the information matrix determinant, for a total of 20,000 measurements ( $N_{x}=200$ along the domain, and $N_{t}=100$ in time). The three curves stand for an increasing number of parameters, $N_{P}=6,9$, and 12, which correspond respectively to $N_{k}=4,7$, and 10 , plus the two end values of thermal conductivity that are filtered from the expansion. Clearly, the gradual increase on the number of parameters markedly decreases the value of the determinant, as illustrated by their values at the end of the time scale, $7.8 \times 10^{-12}, 6.0 \times 10^{-24}$, and $1.0 \times 10^{-38}$, 
respectively, for $N_{P}=6,9$, and 12. Therefore, it has been observed, as expected, that increasing the number of parameters markedly affects the conditioning of the estimation procedure.

Figure $5 b$ presents the information matrix determinant for the case of $N_{P}=9$ parameters, but with a variable number of equally spaced measurements along the domain $\left(N_{x}=200,100,50\right.$, and 5, from top to bottom). The lowest value of $N_{x}$ has been considered to inspect the possibility of employing traditional temperature measurement techniques, such as thermocouples, while the higher values represent a thermographic type of temperature measurement. The determinant of the sensitivity matrix decreases by reducing the number of measurements along the domain $\left(61 \times 10^{-24}, 1.4 \times 10^{-26}, 3.4 \times 10^{-29}\right.$, and $3.3 \times 10^{-38}$, for $N_{x}=200,100,50$, and 5, respectively).

Figure $5 c$ presents the behavior of the determinant of the information matrix (for the uppermost curve in Figure $5 b$ ), with a fixed number of measurements along the domain $\left(N_{x}=200\right)$ and a fixed frequency of measurements $\left(\Delta t=5 \times 10^{-4}\right)$, for the case involving the estimation of nine parameters $\left(N_{P}=9\right)$. We may observe more clearly in this figure the effect of increasing the number of measurements in time $\left(N_{t}=20,50\right.$, and 100), which results in determinants of about the same order of magnitude $\left(1.7 \times 10^{-25}, 2.7 \times 10^{-24}\right.$, and $\left.6.0 \times 10^{-24}\right)$. Hence, the variation on the number of measurements in time within this range does not have a marked effect on the conditioning of the estimation procedure.

The simulated experimental data were generated with standard deviation equal to $1 \%$ of the direct problem temperature values as computed with $N=100$ terms in the temperature expansion, $M=100$ terms in the original eigenfunction expansion, and $N_{k}=20$ terms in both coefficient expansions, $k(x)$ and $w(x)$. The subsequent inverse analysis was performed using just $N=M=15$ terms in both the temperature and eigenfunction expansions, in order to avoid the so-called inverse crime [36]. For the known coefficient, $w(x)$, we have maintained $N_{k}=20$ terms in the expansion, which warrant a converged representation to four significant digits. Based on the information matrix analysis shown above, in solving the inverse problem we then adopted $N_{k}=7$ terms in the thermal conductivity expansion $\left(N_{P}=9\right.$ parameters), $N_{x}=200$ spatial measurements, and $N_{t}=20$ measurements in time.

A relevant aspect in the use of the eigenfunction expansion coefficients as a parameter estimation procedure is the definition of maximum and minimum values for the coefficients to be estimated from the corresponding maximum and minimum values of the thermal conductivity, $k_{\max }$ and $k_{\min }$

The parameterized form of the thermal conductivity used in this application is given by

$$
k(x)=\left(\frac{k_{x=L}-k_{x=0}}{L}\right) x+k_{x=0}+\sum_{k=1}^{N_{k}} \bar{k}_{k} \widetilde{\Gamma}_{k}(x)
$$

which can be rewritten as

$$
\sum_{k=1}^{N_{k}} \bar{k}_{k} \widetilde{\Gamma}_{k}(x)=k(x)-\left(\frac{k_{x=L}-k_{x=0}}{L}\right) x-k_{x=0}
$$


Operating with $\int_{0}^{L} \widetilde{\Gamma}_{i}(x)-\mathrm{dx}$ on both sides of the above equation, we have

$$
\bar{k}_{i}=\int_{0}^{L} \widetilde{\Gamma}_{i}(x) k(x) d x-\left(\frac{k_{x=L}-k_{x=0}}{L}\right) \bar{g}_{i}-k_{x=0} \bar{f}_{i}
$$

where

$$
\begin{aligned}
& \bar{g}_{i}=\int_{0}^{L} x \widetilde{\Gamma}_{i}(x) d x \\
& \bar{f}_{i}=\int_{0}^{L} \widetilde{\Gamma}_{i}(x) d x
\end{aligned}
$$

Thus, for a bounding maximum or minimum $k(x), k_{b}=k_{\min }$ or $k_{b}=k_{\max }$, respectively, we have

$$
\bar{k}_{i, b}=\left(k_{b}-k_{x=0}\right) \bar{f}_{i}-\left(\frac{k_{x=L}-k_{x=0}}{L}\right) \bar{g}_{i}
$$

Since the values of the thermal conductivity at the boundaries are not known $a$ priori, to either maximize or minimize the values of the transformed coefficients in Eq. (32) we need to take into consideration the signs of the coefficients $\bar{g}_{i}$ and $\bar{f}_{i}$. Thus, from the analysis of the expression above, and the specific forms of the transformed quantities, $\bar{g}_{i}$ and $\bar{f}_{i}$ for odd or even indices, one may get conservative upper and lower limits for the expansion coefficients, $\bar{k}_{i \text {,max }}$ and $\bar{k}_{i \text {,min }}$, in the form

For $i=\operatorname{odd} \rightarrow\left(k_{x=0}=k_{x=L}=k_{\min } ; k_{b}=k_{\max }\right)$ :

$$
\begin{aligned}
\bar{k}_{i, \text { max }} & =\frac{2 \sqrt{2}\left(k_{\max }-k_{\min }\right)}{i \pi \sqrt{1 / L}} \\
\bar{k}_{i, \text { min }} & =-\frac{2 \sqrt{2}\left(k_{\max }-k_{\min }\right)}{i \pi \sqrt{1 / L}}
\end{aligned}
$$

For $i=$ even $\rightarrow\left(k_{x=0}=k_{\min } ; k_{x=L}=k_{\max }\right)$

$$
\bar{k}_{i, \max }=\frac{2\left(k_{\max }-k_{\min }\right)}{i \pi \sqrt{1 / L}}
$$

For $i=$ even $\rightarrow\left(k_{x=L}=k_{\min } ; k_{x=0}=k_{\max }\right)$

$$
\bar{k}_{i, \min }=\frac{-\sqrt{2}\left(k_{\max }-k_{\min }\right)}{i \pi \sqrt{1 / L}}
$$

The parameters were estimated using the Metropolis-Hastings algorithm as described in the previous section. To estimate the maximum and minimum ranges for each parameter, we have conservatively adopted as upper limit the filler conductivity, $k_{\max }(x)=k_{d}$, and as lower limit the matrix conductivity $k_{\min }(x)=k_{m}$. Alternatively, the theoretical models previously discussed could have been used to narrow the interval $\left[k_{\min }, k_{\max }\right]$, but at the present stage of tools demonstration, we have 
preferred to employ the wider range. As initial estimate for the coefficient $k(x)$. we have chosen to consider a constant function given by the average value of the coefficient in the range defined above by the upper and lower bounds, $k_{\text {ini }}(x)=\left[k_{\max }(x)+k_{\min }(x)\right] / 2$.

The parameters to be estimated are given by Eq. (28a). Thus, the first two parameters, $k_{x=0}$ and $k_{x=L}$, have their maximum, minimum, and initial values given directly by the choices $k_{\max }(x), k_{\min }(x), k_{\text {ini }}(x)$ evaluated at, respectively, $x=0$ and $x=L$. The other parameters related to the thermal conductivity expansion coefficients have their maximum and minimum values determined as shown in Eqs. (33) and (34), and their initial values are taken equal to the average value between the maximum and minimum parameters obtained. The step adopted in the search procedure used in the generation of candidates within the minimum-maximum parameters range was $20 \%$ of the exact value of the parameter. Table 2 shows the maximum, minimum, the initial search step, and the limiting upper and lower values for the nine parameters to be estimated.

Five illustrative cases were analyzed, corresponding to different $a$ priori information provided. In case 1, a noninformative uniform prior was adopted, while in case 2 the prior was in the form of a normal distribution with mean given by the Lewis and Nielsen thermal conductivity value, with a fairly large $40 \%$ standard deviation. In case 3, a normal prior distribution with mean given by Lewis and Nielsen's correlation was used as in case 2, but with as much as $80 \%$ standard deviation. Cases 4 and 5 deal with the use of a normal prior distribution with mean now given by Maxwell's correlation with $40 \%$ and $80 \%$ standard deviations, respectively, which in principle provide poor prior information for larger concentrations.

Case 1 is expected to provide the worst estimates, since no a priori information is being provided. Cases 2 and 3 employ a Gaussian prior extracted from the same correlation employed in the simulated data, but with reasonably large values of the standard deviation, to challenge the algorithm to recover the thermal conductivity function. Nevertheless, it should be recalled that since the inverse crime has been avoided, the present approach is not expected to exactly recover the same parameters employed to generate the simulated data. Cases 4 and 5 further challenge the inverse analysis approach, by providing a Gaussian prior based on another model (Maxwell) than that employed in generating the experimental data. For lower concentrations

Table 2. Exact values, initial guesses, search step, and search limits for inverse analysis

\begin{tabular}{lcccrr}
\hline Parameter & Exact & Initial guess & Step & $k_{\text {min }}$ & $k_{\text {max }}$ \\
\hline$k_{x=0}$ & 1.0072 & 18.27 & 0.201 & 0.545 & 36 \\
$k_{x=L}$ & 4.2070 & 18.27 & 0.841 & 0.545 & 36 \\
$\bar{k}_{1}$ & 1.0066 & 0 & 0.201 & -31.921 & 31.921 \\
$\bar{k}_{2}$ & 0.01874 & 0 & 0.00375 & -7.980 & 7.980 \\
$\bar{k}_{3}$ & -0.2592 & 0 & 0.0518 & -10.640 & 10.640 \\
$\bar{k}_{4}$ & -0.2441 & 0 & 0.0488 & -3.990 & 3.990 \\
$\bar{k}_{5}$ & -0.1218 & 0 & 0.0244 & -6.384 & 6.384 \\
$\bar{k}_{6}$ & -0.009845 & 0 & 0.00197 & -2.660 & 2.660 \\
$\bar{k}_{7}$ & 0.04450 & 0 & 0.00890 & -4.560 & 4.560 \\
\hline
\end{tabular}


the two models are expected to provide results with reasonable agreement, but with increasing deviations for larger concentrations (22\% deviation as from Figure $3 c$ ).

Assuming a burn-in period of 10,000 states in the Markov chains, and a total of 50,000 states in the whole chains, estimates of the parameters for each case were obtained given by the sample average of the remaining 40,000 states. Table 3 summarizes the estimates found for each parameter and the confidence intervals for a degree of confidence of $95 \%$ for all five cases examined. Clearly, case 1 provides the worst set of results, with very wide confidence intervals, sometimes not even enclosing the exact values, such as for the coefficient $\bar{k}_{6}$. The uniform distribution also performs poorly because the provided bounding limits lead to a very wide interval, which could be at least narrowed by more appropriate information on the function to be estimated, for instance, employing limiting models such as the parallel, series and geometric mean conduction models. As expected, cases 2 and 3 perform quite well, even with the large value of the standard deviation for case 3 , which results in larger confidence intervals for the estimated parameters. In cases 4 and 5 the algorithm still manages to correct the function behavior, by providing reasonable estimates for the parameters, which will be even clearer in the graphical comparisons to follow. Nevertheless, one aspect should be clarified, with respect to the parameter values provided via the chosen Maxwell model as a priori distribution. Although the two thermal conductivity models (Lewis and Nielsen, and Maxwell) are locally different at most by about $22 \%$, after performing the integral transformation to express the two functions in terms of eigenfunction expansions, the deviations between the two sets of expansion coefficients can be much larger, even of the order of $300 \%$. This aspect is not immediately evident from the comparative behavior between the two functions, Figure $3 b$, but it is certainly the main reason for the differences in performance of the inverse analysis in such cases.

Figure $6 a$ shows the upper and lower bounds, the initial guess, and the thermal conductivity function to be recovered in the inverse analysis performed. Figures $6 b-$ $6 f$ show the estimated thermal conductivities in comparison to the exact function, for each of the five cases considered here, based on the estimation of the nine parameters shown in Table 3. These figures confirm the above observations based on Table 3. One may observe the oscillations in the recovered thermal conductivity for the more difficult case involving noninformative uniform prior of case 1 (Figure 6b), as a consequence of the poor estimation of the coefficients in the eigenfunction expansion. Case 2 (Figure $6 c$ ) results in the most accurate estimation obtained, and the increase on the standard deviation to $80 \%$ in the prior (case 3 ) promotes only slight deviations on the estimated thermal conductivity (Figure $6 d$ ). For the last two cases with the prior given from Maxwelĺs correlation, one may notice the corrective action of the algorithm, which distorts the initial guess profile to approach that provided by the exact model, with slightly better agreement for the case with $80 \%$ of standard deviation (see Figures $6 e$ and $6 f$ ). It appears that the higher standard deviation in case 5 ends up by providing more freedom to the algorithm and thus allows a somehow more effective correction of the adopted priori based on another model, different from the one employed to generate the experimental data, in comparison with the more restrictive priori with a smaller standard deviation of case 4 .

Finally, Figures 7 and 8 illustrate the Markov chain evolutions along the 50,000 states for all the nine parameters, in cases 1 and 2, respectively. From 
Table 3. Estimated parameter values with 50,000 states in Markov chains (neglecting first 10,000 states for chain burn-in) and corresponding $95 \%$ confidence intervals $^{a}$

\begin{tabular}{|c|c|c|c|c|c|}
\hline $\mathrm{P}$ & Case 1 & Case 2 & Case 3 & Case 4 & Case 5 \\
\hline$k_{x=0}$ & $1.426[0.630,2.775]$ & $1.075[0.7041,1.487]$ & $1.163[0.655,1.813]$ & $1.010[0.684,1.390]$ & $1.063[0.644,1.569]$ \\
\hline$k_{x=L}$ & $6.921[1.950,14.327]$ & $4.300[2.483,6.424]$ & $4.646[2.199,7.837]$ & $3.979[2.505,5.576]$ & $4.538[2.373,7.102]$ \\
\hline $\bar{k}_{1}$ & $0.307[-2.189,3.207]$ & $0.957[0.356,1.610]$ & $0.841[-0.279,1.908]$ & $0.757[0.249,1.261]$ & $0.655[-0.226,1.510]$ \\
\hline $\bar{k}_{2}$ & $0.328[-0.068,0.586]$ & $0.0185[0.0034,0.033]$ & $0.018[-0.016,0.049]$ & $0.0747[0.029,0.121]$ & $0.067[-0.0013,0.147]$ \\
\hline $\bar{k}_{3}$ & $-0.336[-1.238,0.663]$ & $-0.270[-0.452,-0.086]$ & $-0.293[-0.625,0.048]$ & $-0.146[-0.248,-0.047]$ & $-0.169[-0.353,0.0223]$ \\
\hline $\bar{k}_{4}$ & $-0.487[-1.816,0.706]$ & $-0.244[-0.419,-0.0582]$ & $-0.280[-0.596,0.044]$ & $-0.180[-0.309,-0.05]$ & $-0.204[-0.437,0.030]$ \\
\hline $\bar{k}_{5}$ & $-0.511[-1.305,0.288]$ & $-0.1216[-0.214,-0.0248]$ & $-0.113[-0.294,0.0782]$ & $-0.107[-0.187,-0.029]$ & $-0.116[-0.274,0.056]$ \\
\hline $\bar{k}_{6}$ & $0.154[0.029,0.271]$ & $-0.0101[-0.017,-0.0024]$ & $-0.00976[-0.027,0.006]$ & $-0.0388[-0.064,-0.016]$ & $-0.031[-0.090,0.023]$ \\
\hline $\bar{k}_{7}$ & $0.202[-0.167,0.625]$ & $0.0453[0.013,0.0795]$ & $0.0425[-0.024,0.113]$ & $0.0088[0.0021,0.016]$ & $0.0089[-0.005,0.023]$ \\
\hline
\end{tabular}

${ }^{a}$ Case 1, uniform prior; case 2, normal prior from Lewis and Nielsen with $40 \%$ standard deviation; case 3, normal prior from Lewis and Nielsen with $80 \%$ standard deviation; case 4, normal prior from Maxwell with 40\% standard deviation; case 5, normal prior from Maxwell with $80 \%$ standard deviation. 


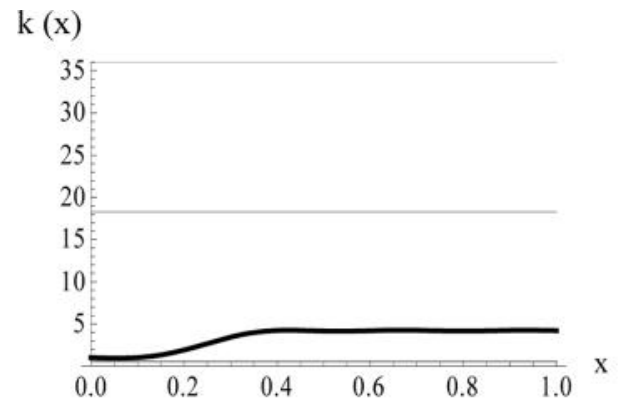

(a) Expansion, initial guess and limits.

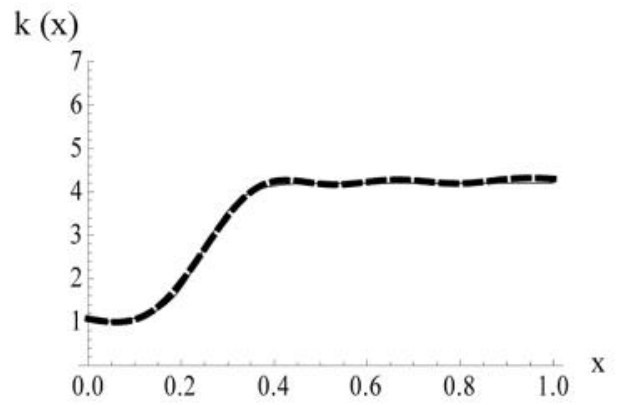

(c) Case 2

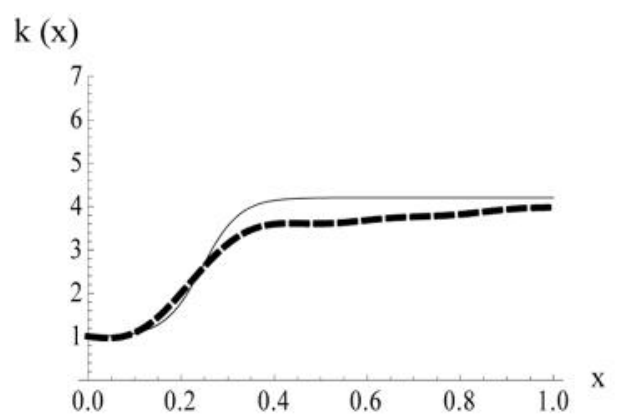

(e) Case 4

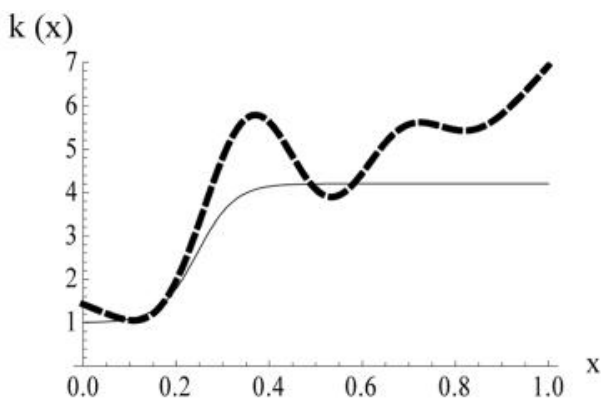

(b) Case 1

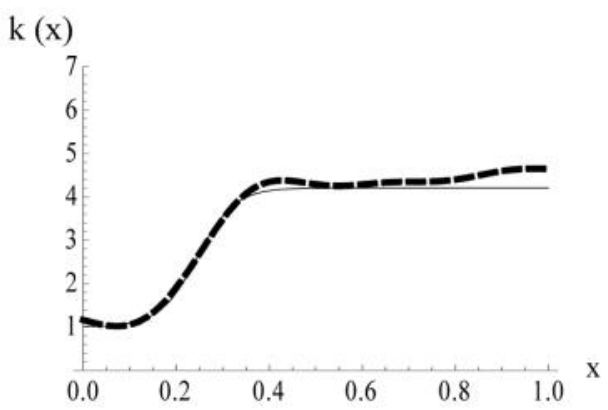

(d) Case 3

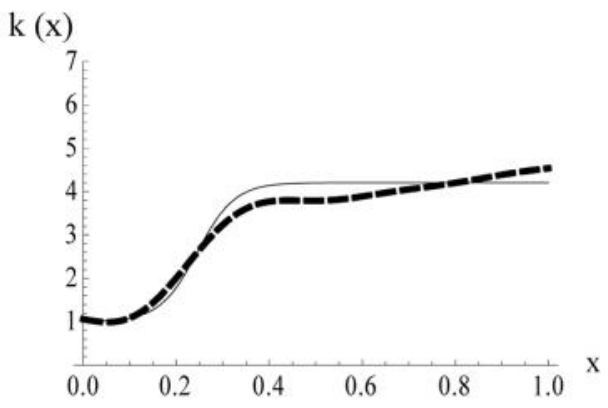

(f) Case 5

Figure 6. (a) Exact and expanded $k(x)$, initial guess and search limits provided, and $(b-f)$ comparison of exact (solid) and estimated (dashed) thermal conductivity functions in all five cases considered.

Figure 7, related to the noninformative uniform prior, and in light of the very wide upper and lower bounds proposed here, one may notice the significant large amplitudes in the chain oscillations, which is not yet fully converged for some of the parameters. On the other hand, the chains obtained with the normal prior provided from Lewis and Nielsen's model, which are presented in Figure 8, have oscillations with small amplitudes and are fully converged within this range of 50,000 states. Also, although not illustrated here due to space limitations, increasing the uncertainty of the simulated temperature measurements essentially increases the amplitudes 

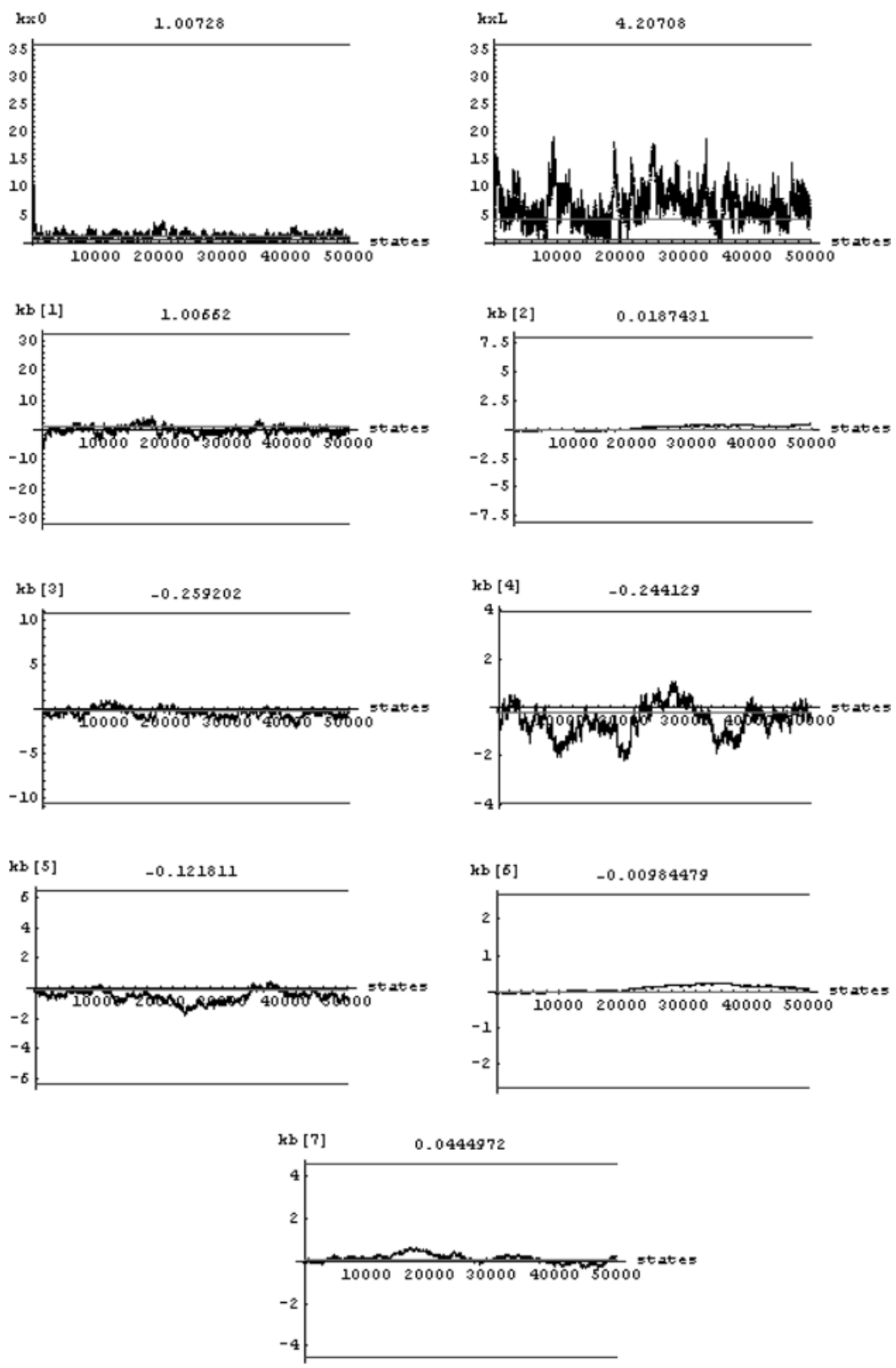

Figure 7. Markov chain evolutions of the nine parameters for case 1 .

of the Markov chains convergence patterns, basically yielding larger confidence intervals for the estimated parameters. However, we note that the simulated measurement errors used above are indeed much larger than those usually observed in measurements with infrared cameras. 

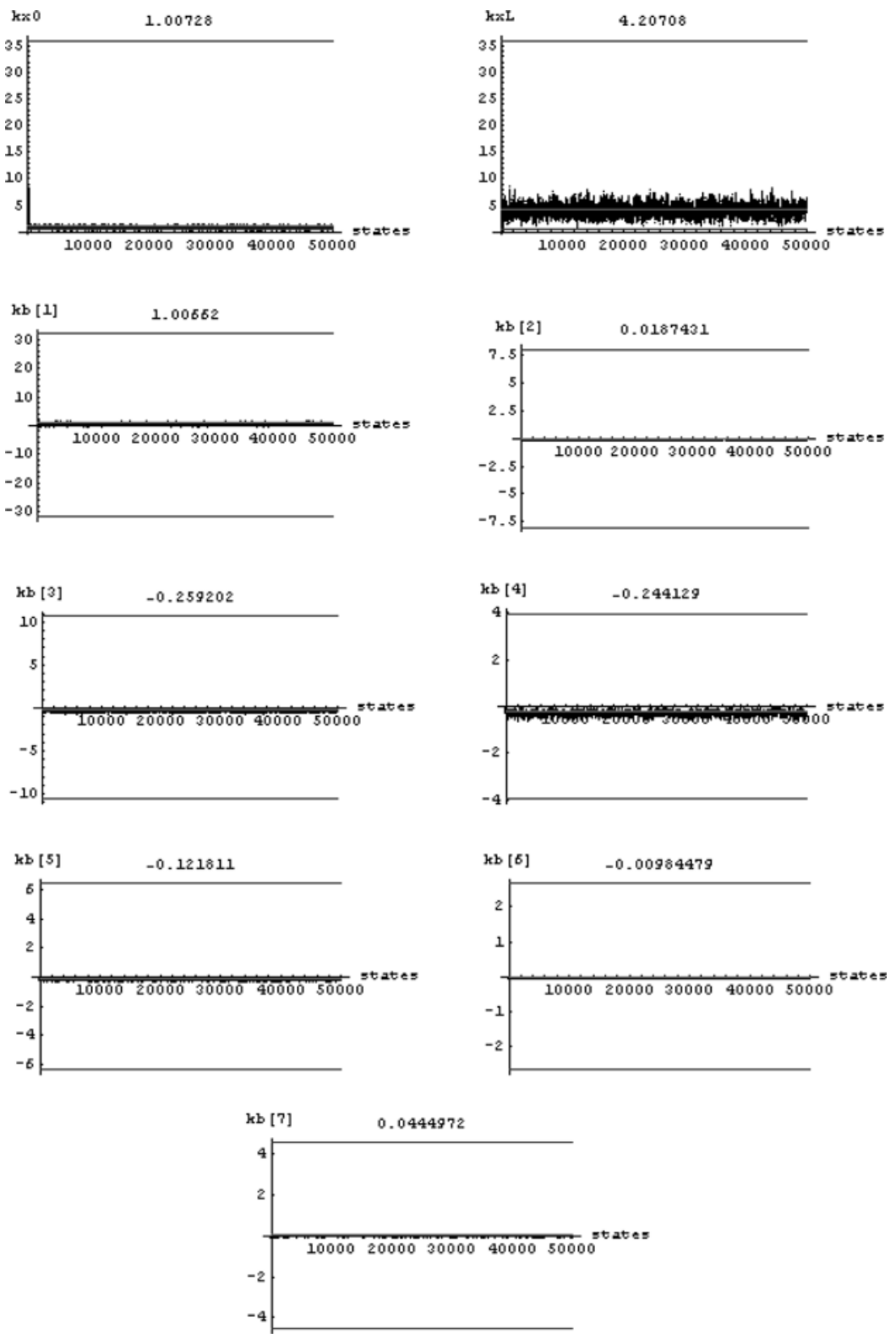

Figure 8. Markov chain evolutions of the nine parameters for case 2.

\section{CONCLUSIONS}

The combined use of integral transforms and Bayesian inference was demonstrated for the inverse problem of estimating space-variable thermal conductivity in two-phase dispersed systems, undergoing a transient one-dimensional heat conduction process. The direct problem solution was obtained analytically with the 
classical integral transform method, while the related eigenvalue problem, which carries the information on the medium heterogeneities, was solved with the generalized integral transform technique (GITT).

The inverse problem solution was based on the Markov chain Monte Carlo method. The Metropolis-Hastings algorithm was employed for the sampling procedure, all implemented in the Mathematica symbolic computation platform. Instead of seeking the function estimation in the form of a set of local values of the thermal conductivity, an alternative approach was proposed in this work, using an eigenfunction expansion of the thermal conductivity itself. Both Gaussian and noninformative uniform distributions were used as priors for the solution of the present inverse problem, obtained with simulated experimental data. Priors obtained from alternative theoretical relations for the thermal conductivity, which yield different predictions than those employed in the simulated experimental data, were also employed in the algorithm, in order to demonstrate robustness of the inverse analysis.

The results obtained in this work reveal that the proposed inverse analysis approach is robust with respect to measurement errors and capable of providing accurate results even for prior distributions quite different from the exact one. Such inverse analysis will now allow for the verification of the adequacy of the existing models or even for their adaptation and formulation of novel predictive expressions, for a wide variety of geometric and materials configurations, filler concentration distributions, and other relevant variables. In addition, for more complex systems, such as hybrid or multicomponent dispersed systems, when models are even not yet readily available or reliable, the present inverse analysis approach can provide accurate estimation of the thermophysical properties variation.

\section{REFERENCES}

1. C. Zweben, Advances in Composite Materials for Thermal Management in Electronic Packaging, J. Minerals, Metals Materials Soc., vol. 50, pp. 47-51, 1998.

2. D. M. Bigg, Thermal Conductivity of Heterophase Polymer Compositions, Adv. Polymer Sci., vol. 119, pp. 1-30, 1995.

3. Y. Yang, Thermal Conductivity, in J. E. Mark (ed.), Physical Properties of Polymers Handbook, Part III, Chap. 10, pp. 155-163, 2007.

4. R. C. Progelhof, J. L. Throne, and R. R. Ruetsch, Methods for Predicting the Thermal Conductivity of Composite Systems: A Review, Polymer Eng. Sci., vol. 16, pp. 615-625, 1976.

5. I. H. Tavman, Thermal and Mechanical Properties of Aluminum Powder-Filled High-Density Polyethylene Composites, J. Appl. Polymer Sci., vol. 62, pp. 2161-2167, 1996.

6. I. H. Tavman and H. Akinci, Transverse Thermal Conductivity of Fiber Reinforced Polymer Composites, Int. Commun. Heat Mass Transfer, vol. 27, pp. 253-261, 2000.

7. N. M. Sofian, M. Rusu, R. Neagu, and E. Neagu, Metal Powder-Filled Polyethylene Composites. V. Thermal Properties, J. Thermoplastic Composite Mater., vol. 14, pp. 2033, 2001.

8. Ye. P. Mamunya, V. V. Davydenko, P. Pissis, and E. V. Lebedev, Electrical and Thermal Conductivity of Polymers Filled with Metal Powders, Eur. Polymer J., vol. 38, pp. 1887-1897, 2002. 
9. D. Kumlutas, I. H. Tavman, and M. T. Çoban, Thermal Conductivity of Particle Filled Polyethylene Composite Materials, Composite Sci. Technol., vol. 63, pp. 113-117, 2003.

10. F. Danes, B. Garnier, and T. Dupuis, Predicting, Measuring and Tailoring the Transverse Thermal Conductivity of Composites from Polymer Matrix and Metal Filler, Int. J. Thermophys., vol. 24, pp. 771-784, 2003.

11. A. Boudenne, L. Ibos, M. Fois, E. Gehin, and J. C. Majeste, Thermophysical Properties of Polypropylene/Aluminum Composites, J. Polymer Sci., B, Polymer Phys., vol. 48, pp. 722-732, 2004.

12. B. Weidenfeller, M. Hofer, and F. R. Schilling, Thermal Conductivity, Thermal Diffusivity, and Specific Heat Capacity of Particle Filled Polypropylene, Composites A, vol. 35, pp. 423-429, 2004.

13. A. Boudenne, L. Ibos, M. Fois, J. C. Majeste, and E. Gehin, Electrical and Thermal Behavior of Polypropylene Filled with Copper Particles, Composites A, vol. 36, pp. 1545-1554, 2005.

14. D. Kumlutas and I. H. Tavman, A Numerical and Experimental Study on Thermal Conductivity of Particle Filled Polymer Composites, J. Thermoplastic Composite Mater., vol. 19, pp. 441-455, 2006.

15. H. Serkan Tekce, D. Kumlutas, and I. H. Tavman, Effect of Particle Shape on Thermal Conductivity of Copper Reinforced Polymer Composites, J. Reinforced Plastics Composites, vol. 26, pp. 113-121, 2007.

16. D. Lesnic, L. Elliot, D. B. Ingham, B. Clennell, and R. J. Knioe, The Identification of the Piecewise Homogeneous Thermal Conductivity of Conductors Subjected to a Heat Flow Test, Int. J. Heat Mass Transfer, vol. 42, pp. 143-152, 1999.

17. E. Divo, A. Kassab, and F. Rodriguez, Characterization of Space Dependent Thermal Conductivity with a BEM-Based Genetic Algorithm, Numer. Heat Transfer A, vol. 37, pp. 845-875, 2000.

18. F. A. Rodrigues, H. R. B. Orlande, and G. S. Dulikravich, Simultaneous Estimation of Spatially Dependent Diffusion Coefficient and Source Term in a Nonlinear 1D Diffusion Problem, Math. Comput. Simulation, vol. 66, pp. 409-424, 2004.

19. M. R. Golbahar Haghighi, M. Eghtesad, P. Malekzadeh, and D. S. Necsulescu, Two-Dimensional Inverse Heat Transfer Analysis of Functionally Graded Materials in Estimating Time-Dependent Surface Heat Flux, Numer. Heat Transfer A, vol. 54, pp. 744-762, 2008.

20. S. H. Lin, Transient Conduction in Heterogeneous Media, Int. Commun. Heat Mass Transfer, vol. 10, pp. 165-174, 1992.

21. E. Divo and A. Kassab, Generalized Boundary Integral Equation for Transient Heat Conduction in Heterogeneous Media, J. Thermophys. Heat Transfer, vol. 12, p. 364, 1998.

22. B. Chen, L. Tong, Y. Gu, H. Zhang, and O. Ochoa, Transient Heat Transfer Analysis of Functionally Graded Materials Using Adaptive Precise Time Integration and Graded Finite Elements, Numer. Heat Transfer B, vol. 45, pp. 181-200, 2004.

23. J. Fang, G. F. Zhao, J. Zhao, and A. Parriaux, On the Truly Meshless Solution of Heat Conduction Problems in Heterogeneous Media, Numer. Heat Transfer B, vol. 55, pp. 1-13, 2009.

24. M. D. Mikhailov and M. N. Ozisik, Unified Analysis and Solutions of Heat and Mass Diffusion, Wiley, Newyork, 1984; Dover, Newyork, 1994.

25. R. M. Cotta, Integral Transforms in Computational Heat and Fluid Flow, CRC Press, Boca Raton, FL, 1993.

26. R. M. Cotta and M. D. Mikhailov, Heat Conduction: Lumped Analysis, Integral Transforms, Symbolic Computation, Wiley-Interscience, New York, 1997.

27. R. M. Cotta, The Integral Transform Method in Thermal and Fluids Sciences and Engineering, Begell House, New York, 1998. 
28. R. M. Cotta and M. D. Mikhailov, Hybrid Methods and Symbolic Computations, in W. J. Minkowycz, E. M. Sparrow, and J. Y. Murthy (eds.), Handbook of Numerical Heat Transfer, 2nd ed., pp. 493-522, Wiley, New York, 2006.

29. M. D. Mikhailov and R. M. Cotta, Integral Transform Method for Eigenvalue Problems, Commun. Numer. Meth. Eng., vol. 10, pp. 827-835, 1994.

30. L. A. Sphaier and R. M. Cotta, Integral Transform Analysis of Multidimensional Eigenvalue Problems within Irregular Domains, Numer. Heat Transfer B, vol. 38, pp. 157-175, 2000.

31. S. C. Hirata, B. Goyeau, D. Gobin, and R. M. Cotta, Stability of Natural Convection in Composite Enclosures Using Integral Transforms, Numer. Heat Transfer B, vol. 50, pp. 409-424, 2006.

32. C. P. Naveira-Cotta, R. M. Cotta, H. R. B. Orlande, and O. Fudym, Eigenfunction Expansions for Transient Diffusion in Heterogeneous Media, Int. J. Heat Mass Transfer, vol. 52, pp. 5029-5039, 2009.

33. S. Wolfram, The Mathematica Book, version 5.2, Cambridge-Wolfram Media, UrbanaChampaign, IL, 2005.

34. J. Beck and K. Arnold, Parameter Estimation in Engineering and Science, Wiley Interscience, New York, 1977.

35. M. N. Ozisik and H. R. B. Orlande, Inverse Heat Transfer: Fundamentals and Applications, Taylor \& Francis, New York, 2000.

36. J. Kaipio and E. Somersalo, Statistical and Computational Inverse Problems, Springer-Verlag, Berlin, 2004.

37. P. Lee, Bayesian Statistics, Oxford University Press, London, 2004.

38. D. Gamerman and H. F. Lopes, Markov Chain Monte Carlo: Stochastic Simulation for Bayesian Inference, 2nd ed., Chapman \& Hall/CRC, Boca Raton, FL, 2006.

39. H. S. Migon and D. Gamerman, Statistical Inference: An Integrated Approach, Arnold, Oxford, London, New York, 1999.

40. H. R. B. Orlande, M. J. Colaço, and G. S. Dulikravich, Approximation of the Likelihood Function in the Bayesian Technique for the Solution of Inverse Problems, Inverse Prob. Sci. Eng., vol. 16, pp. 677-692, 2008.

41. O. Fudym, H. R. B. Orlande, M. Bamford, and J. C. Batsale, Bayesian Approach for Thermal Diffusivity Mapping from Infrared Images Processing with Spatially Random Heat Pulse Heating, J. Phys. Conf. Ser. (online), vol. 135, pp. 12-42, 2008.

42. N. Metropolis, A. W. Rosenbluth, M. N. Rosenbluth, A. H. Teller, and E. Teller, Equations of State Calculations by Fast Computating Machines, J. Chemi. Phys., vol. 21, pp. 1087-1092, 1953.

43. W. K. Hastings, Monte Carlo Sampling Methods Using Markov Chains and Their Applications, Biometrika, vol. 57, pp. 97-109, 1970.

44. A. A. Barker, Monte Carlo Calculation of the Radial Distribution Functions for a Proton-Electron Plasma, Austral. J. Phys., vol. 18, pp. 119-133, 1965.

45. P. H. Peskun, Optimum Monte Carlo Sampling Using Markov Chain, Biometrika, vol. 60, pp. 607-612, 1973.

46. J. C. Maxwell, A Treatise on Electricity and Magnetism, Dover, New York, 1954.

47. T. Lewis and L. Nielsen, Dynamic Mechanical Properties of Particulate-Filled Polymers, J. Appl. Polymer Sci., vol. 14, pp. 1449-1471, 1970.

48. Y. Agari and T. Uno, Estimation on Thermal Conductivities of Filled Polymers, J. Appl. Polymer Sci., vol. 32, pp. 5705-5712, 1986. 